\title{
Ensemble X-ray variability of active galactic nuclei from serendipitous source catalogues ${ }^{\star}$
}

\author{
F. Vagnetti ${ }^{1}$, S. Turriziani ${ }^{1, \star \star}$, and D. Trevese ${ }^{2}$ \\ 1 Dipartimento di Fisica, Università di Roma “Tor Vergata”, Via della Ricerca Scientifica 1, 00133 Roma, Italy \\ e-mail: fausto.vagnetti@roma2.infn.it \\ 2 Dipartimento di Fisica, Università di Roma "La Sapienza", Piazzale Aldo Moro 2, 00185 Roma, Italy \\ Received 12 September 2011 / Accepted 20 October 2011
}

ABSTRACT

\begin{abstract}
Context. The X-ray variability of the active galactic nuclei (AGN) has been most often investigated with studies of individual, nearby sources, and only a few ensemble analyses have been applied to large samples in wide ranges of luminosity and redshift.

Aims. We aim to determine the ensemble variability properties of two serendipitously selected AGN samples extracted from the catalogues of XMM-Newton and Swift, with redshift between $\sim 0.2$ and $\sim 4.5$, and X-ray luminosities, in the $0.5-4.5 \mathrm{keV}$ band, between $\sim 10^{43} \mathrm{erg} / \mathrm{s}$ and $\sim 10^{46} \mathrm{erg} / \mathrm{s}$.

Methods. We used the structure function (SF), which operates in the time domain, and allows for an ensemble analysis even when only a few observations are available for individual sources and the power spectral density (PSD) cannot be derived. The SF is also more appropriate than fractional variability and excess variance, because these parameters are biased by the duration of the monitoring time interval in the rest-frame, and therefore by cosmological time dilation.

Results. We find statistically consistent results for the two samples, with the SF described by a power law of the time lag, approximately as $S F \propto \tau^{0.1}$. We do not find evidence of the break in the SF, at variance with the case of lower luminosity AGNs. We confirm a strong anti-correlation of the variability with X-ray luminosity, accompanied by a change of the slope of the SF. We find evidence in support of a weak, intrinsic, average increase of X-ray variability with redshift.

Conclusions. The change of amplitude and slope of the SF with X-ray luminosity provides new constraints on both single oscillator models and multiple subunit models of variability.
\end{abstract}

Key words. surveys - galaxies: active - quasars: general - X-rays: galaxies

\section{Introduction}

Active galactic nuclei (AGN) typically show flux variability in all wavebands and on different timescales from minutes to years. This behaviour has been widely used to constrain the size and location of the emission regions and to obtain information on the emission mechanisms as well as the processes that cause the variability itself.

In addition to the study of individual light curves, ensemble properties of statistical AGN samples have been investigated in the optical/UV band through the use of the structure function (SF) (e.g. Trevese et al. 1994; Cristiani et al. 1996; Vanden Berk et al. 2004), and in the X-rays through the analysis of the fractional variability (Almaini et al. 2000; Manners et al. 2002).

Optical variability has been found to increase with decreasing luminosity (e.g. Cristiani et al. 1996), and with increasing redshift (Giallongo et al. 1991). The average increase with redshift of the amplitude of variability can be explained by the fact that high-redshift sources are observed at a higher restframe frequency, where they are more variable (di Clemente et al. 1996). The stronger variability at higher frequency, in turn, is caused by a hardening of the spectral energy distribution (SED) in the brighter phase, as shown by ensemble analyses of multiband optical photometry of quasar (QSO) samples (Trevese et al. 2001; Trevese \& Vagnetti 2002). More

* Tables 1 and 2 are available in electronic form at http://www . aanda.org

$\star \star$ Visitor at ASI Science Data Center, c/o ESRIN, Via G. Galilei, 00044 Frascati, Italy. recently, Vanden Berk et al. (2004) applied an ensemble analysis to a large sample of 25000 QSOs observed at two epochs only with the Sloan Digital Sky Survey (SDSS). The authors analysed variability as a function of intrinsic luminosity, redshift, rest-frame frequency and time lag between the observations, proposing a weak, intrinsic increase of variability with redshift, in addition to the amount previously explained by the stronger variability at higher rest-frame frequency (di Clemente et al. 1996), although additional analyses have not confirmed this increase (e.g. MacLeod et al. 2010).

In the X-ray domain, variability occurs on shorter time scales than in any other band, and is thought to come from a hot corona close to the central black hole (BH). Most investigations concern the light curves of individual nearby Seyfert 1 AGNs (e.g. Uttley et al. 2002; Uttley \& McHardy 2005). It has been found that low-luminosity AGNs are generally more variable than higher luminosity ones (e.g. Barr \& Mushotzky 1986; Lawrence \& Papadakis 1993; Green et al. 1993; Nandra et al. 1997), and that the variability amplitude is higher on long time scales than on short time scales (e.g. Markowitz \& Edelson 2004). In addition, it has been suggested that variability also increases with redshift (Paolillo et al. 2004).

Proposed variability models include a single coherent oscillator (e.g. Almaini et al. 2000), a superposition of individual flares or spots (e.g. Lehto 1989; Abramowicz et al. 1991; Czerny et al. 2004), variable absorption and/or reflection (e.g. Abrassart \& Czerny 2000; Miniutti \& Fabian 2004; Chevallier et al. 2006).

The relation between X-ray and optical/UV variability may be due to either i) Compton up-scattering in the hot corona of 
optical photons emitted by the disk (Haardt \& Maraschi 1991); or to ii) a reprocessing of X-rays into thermal optical emission by means of irradiation and heating of the accretion disk (CollinSouffrin 1991). In the first case, variations in the optical/UV flux would lead to X-ray variations, and vice versa in the latter case. Cross-correlation analyses of well-sampled X-ray and optical/UV light curves allow us to constrain models for the cause of the variability. The main results obtained so far indicate a cross-correlation between X-ray and UV/optical variations on the timescale of days, and in some cases delays between the two bands have been measured, with both X-rays lagging the UV (e.g. Marshall et al. 2008; Doroshenko et al. 2009), and vice versa (e.g. Shemmer et al. 2001; Arévalo et al. 2009).

Even more insight into the relation between X-ray and opti$\mathrm{cal} / \mathrm{UV}$ variability is given by the analysis of the X-ray/UV ratio and its variability. Vagnetti et al. (2010) have shown that variability of $\alpha_{\mathrm{ox}}{ }^{1}$ increases as a function of time-lag for a sample of serendipitously selected AGNs with simultaneous X-ray and UV measurements. This contributes part of the observed dispersion in the $\alpha_{\mathrm{Ox}}-L_{\mathrm{UV}}$ anti-correlation, while another contribution is given by intrinsic differences among the average values of each AGN.

In the present paper, we present for the first time an ensemble structure function analysis of the variability of AGNs in the X-ray band. We adopt two sets of multi-epoch X-ray measurements extracted from the serendipitous source catalogues of XMM-Newton (Watson et al. 2009) and Swift (Puccetti et al. 2011).

The paper is organised as follows. Section 2 describes the data extracted from the archival catalogues. Section 3 describes the computation of the structure functions, and discusses their shapes, their dependence on black hole mass and bolometric luminosity, as well as on X-ray luminosity and redshift. In Sect. 4 we discuss and summarise the results.

Throughout the paper, we adopt the cosmology $H_{0}=$ $70 \mathrm{~km} \mathrm{~s}^{-1} \mathrm{Mpc}^{-1}, \Omega_{\mathrm{m}}=0.3$, and $\Omega_{\Lambda}=0.7$.

\section{The data}

\subsection{XMM-Newton}

The XMM-Newton Serendipitous Source Catalogue (XMMSSC) (Watson et al. 2009) is a comprehensive catalogue of serendipitous X-ray sources from the XMM-Newton observatory. The version presently available is $2 \mathrm{XMMi}-\mathrm{DR} 3$, the latest incremental update of the second version of the catalogue ${ }^{2}$. It contains source detections drawn from 4953 XMM-Newton EPIC observations made between 2000 February 3 and 2008 October 08; all datasets were publicly available by 2009 October 31, but not all public observations are included in this catalogue. The total area of the catalogue fields is $\sim 814 \mathrm{deg}^{2}$, but taking account of the substantial overlaps between observations, the net sky area covered independently is $\sim 504 \mathrm{deg}^{2}$. The 2XMMi-DR3 catalogue contains 353191 detections (above the processing likelihood threshold of 6), related to 262902 unique X-ray sources, therefore a significant number of sources (41979) have more than one record within the catalogue.

We used the TOPCAT ${ }^{3}$ software to extract the sources with repeated X-ray observations from the 2XMMi-DR3 catalogue and cross-correlated this list with the DR7 edition of the SDSS

\footnotetext{
${ }_{1} \alpha_{\text {ox }} \equiv \log \left(L_{2 \mathrm{keV}} / L_{2500} \AA\right) / \log \left(v_{2 \mathrm{keV}} / v_{2500} \AA\right)$.

2 http://xmmssc-wWw.star.le.ac.uk/Catalogue/

${ }^{3}$ http://www.star.bris.ac.uk/ mbt/topcat/
}

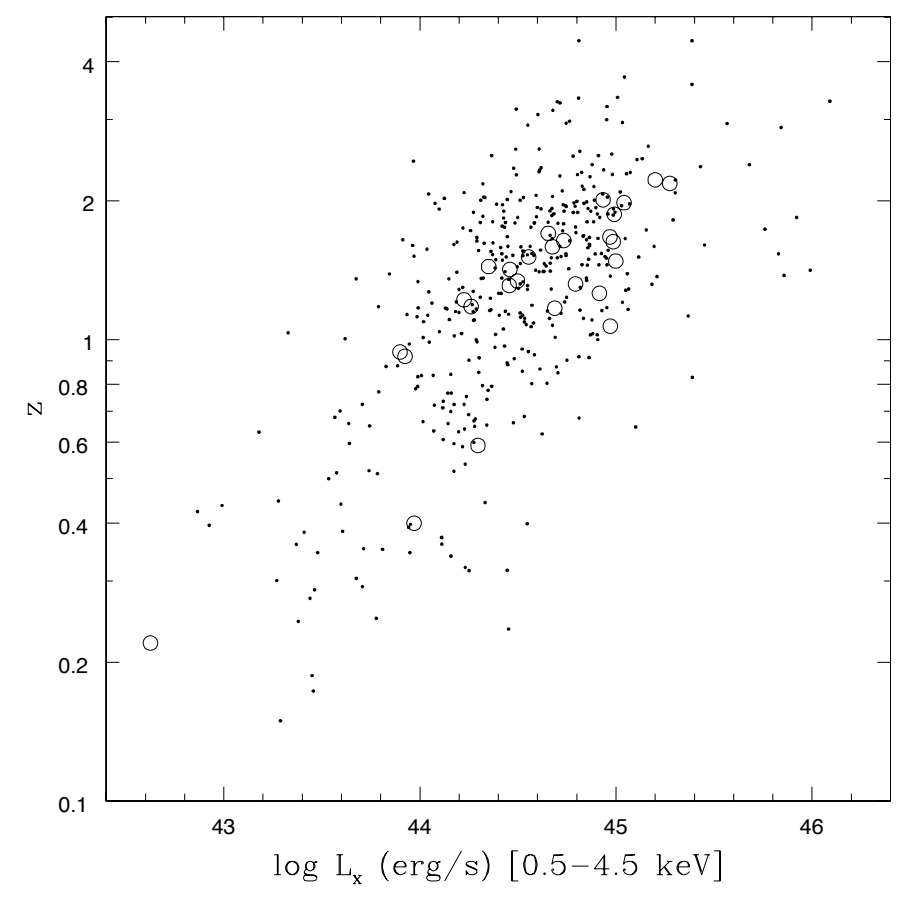

Fig. 1. Distribution of the sources in the $L_{\mathrm{X}}-z$ plane. Dots: XMM-Newton sample; circles: Swift sample.

Quasar Catalogue (Schneider et al. 2010) to obtain redshifts and spectral classifications of the sources. We used a maximum distance of 1.5 arcsec, corresponding to the uncertainty in the X-ray position, resulting in 412 quasars that were observed from 2 to 25 epochs each for a total of 1376 observations. We refer to these sources as the XMM-Newton sample, and report them in Table 1, where Col. 1 corresponds to the source serial number; Col. 2 gives the source name; Col. 3 the redshift; Col. 4 the number of observation epochs for the source; Col. 5 the average log of the X-ray flux in the observed $0.5-4.5 \mathrm{keV}$ band, in erg $\mathrm{cm}^{-2} \mathrm{~s}^{-1}$; Col. 6 the average log of the X-ray luminosity in the $0.5-4.5 \mathrm{keV}$ band, in erg $\mathrm{s}^{-1}$, computed with a photon index $\Gamma=1.8$; and Cols. 7 and 8 the log of the minimum and maximum lag between any two epochs of the light curve in the rest-frame of the source in days.

The sources are shown in the $L_{\mathrm{X}}-z$ plane in Fig. 1 together with the sources of the Swift sample (Sect. 2.2). Here and throughout we adopted the same X-ray band $0.5-4.5 \mathrm{keV}$ for the two samples. For XMMSSC, the flux was directly extracted from the EP9 band of the catalogue, while for the Swift sample the flux was computed from the Swift band $0.3-10 \mathrm{keV}$, adopting a photon index $\Gamma=1.8$.

Typical monitoring times range from months to few years in the rest-frame. Some of the best sampled light curves with 10 or more epochs are shown in Fig. 2, with times in rest-frame days, counted from the initial epoch of each light curve.

\subsection{Swift}

In the context of serendipitous surveys, the Swift satellite provides a unique capability. Although this space observatory is designed to discover gamma-ray bursts (GRB) (Gehrels et al. 2004), it is possible to use individual pointed observations of each GRB to build a large sample of deep X-ray images by stacking the individual exposures. To this purpose, Puccetti et al. (2011) considered all Swift GRB observations 
F. Vagnetti et al.: X-ray variability of AGNs

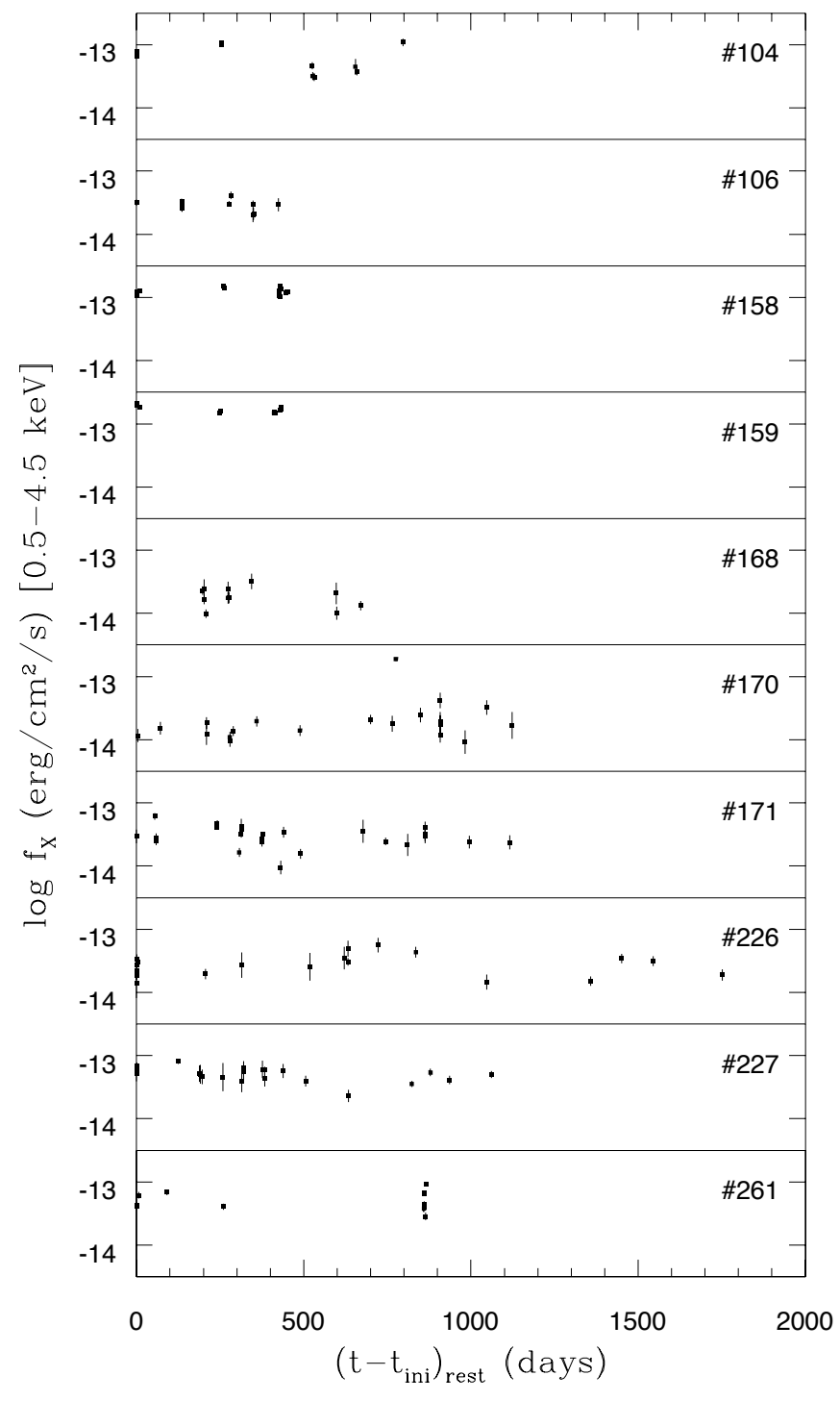

Fig. 2. Some of the best-sampled light curves from the XMM-Newton sample. Times are counted from the initial epoch of each light curve in the rest-frame. Errors, proportional to the inverse square root of the photon count at each epoch, are displayed as 3- $\sigma$ values. Errors are discussed in more detail in Sect. 3. Source numbers from Col. 1 of Table 1 are indicated.

from January 2005 to December 2008, with a total exposure time in the X-ray Telescope (XRT) longer than $10 \mathrm{ks}$. These authors also analysed the XRT $0.5 \mathrm{Ms}$ observation of the Chandra Deep Field South (CDFS) sky region. This set of observations is called the Swift Serendipitous Survey in deep XRT GRB Fields (S3XGF). These 374 images make up an unbiased X-ray survey because GRBs explode at random positions in the sky, and Puccetti et al. (2011) used them to define a well-suited statistical sample of X-ray point sources. The total exposure time of the survey is $36.8 \mathrm{Ms}$, with $\sim 32 \%$ of the fields with more than $100 \mathrm{ks}$ exposure time, and $\sim 28 \%$ with exposure time in the range $50-100 \mathrm{ks}$. The survey covers a total area of $\sim 32.55 \mathrm{deg}^{2}$.

We used the preliminary version S3XGF catalogue, comprising GRB fields observed from January 2005 to June 2007, and cross-correlated it with the DR7 edition of the SDSS Quasar Catalogue (Schneider et al. 2010) to obtain redshifts and spectral classifications.
We found 27 confirmed quasars with sufficient sampling (at least 100 photons in the light curve) to be used in the following $\mathrm{SF}$ analysis. These sources, to which we will refer as the Swift sample, are reported in Table 2, where Col. 1 corresponds to the source serial number; Col. 2 to the source name; Col. 3 gives the redshift; Col. 4 the number of time bins into which we divide the light curve according to the procedure described in the following; Col. 5 the average log of the X-ray flux in the band $0.5-4.5 \mathrm{keV}$, in erg cm $\mathrm{cm}^{-2} \mathrm{~s}^{-1}$; Col. 6 the average log of the X-ray luminosity in the band $0.5-4.5 \mathrm{keV}$, in $\mathrm{erg} \mathrm{s}^{-1}$; Col. 7 the GRB field where the source was observed.

The light curve files extracted from the Swift archive contain sequences of time intervals $\Delta t_{i}$ between $t_{\mathrm{star}, i}$ and $t_{\mathrm{stop}, i}$, in which the telescope was observing, with $n_{i}$ the number of photons detected in each interval. We binned the light curves using a bin size $\Delta t_{\text {bin }}=5 \times 10^{4} \mathrm{~s}$, which is a good compromise to obtain an average number of photons/bin $\gtrsim 10$ and a number of useful bins (i.e., bins with non-zero number of photons) in the light curve $\gtrsim 10$. There is a negligible number of bins with zero photons, however. We assigned an average time $t_{j}$ to each bin $j$ weighted by the number of photons detected in the intervals (or fractions of intervals) $\Delta t_{i}$ overlapped with the bin: $t_{j}=\sum n_{i} t_{i} / \sum n_{i}$, where $t_{i}=\left(t_{\mathrm{star}, i}+t_{\mathrm{stop}, i}\right) / 2$.

Some of the best-sampled light curves are shown in Fig. 3 with times in rest-frame days counted from the initial epoch of each light curve. Typical monitoring times range from some days to a few weeks in the rest-frame, and are therefore complementary to the time scales sampled by XMM-Newton .

The distribution of the Swift sample in the $L_{\mathrm{X}}-z$ plane is shown in Fig. 1 together with the XMM-Newton sample.

\section{The structure function}

The structure function (SF) has the great advantage of working in the time domain, which allows for an ensemble analysis even for extremely poor sampling of individual objects, when the armonic content is completely lost. In this case, the structure function is to be preferred over power spectral density (PSD) analysis (e.g. Hughes et al. 1992; Collier \& Peterson 2001; Favre et al. 2005). The SF was first introduced by Simonetti et al. (1985), and has since been used in various bands, including radio (e.g. Hughes et al. 1992), optical (e.g. Trevese et al. 1994; Kawaguchi et al. 1998; de Vries et al. 2003; Bauer et al. 2009), and X-ray (e.g. Fiore et al. 1998; Brinkmann et al. 2001; Gliozzi et al. 2001; Iyomoto \& Makishima 2001; Zhang et al. 2002).

The SF provides a measure of the mean deviation for data points separated by a time lag $\tau$, and is defined in various ways in the literature. A variant in the definition concerns the use of the average square difference (e.g. Simonetti et al. 1985; Hughes et al. 1992) or the average of the absolute values of the differences (di Clemente et al. 1996). Another variant concerns the use of magnitudes or fluxes: while in the optical the SF is usually defined in terms of magnitude differences, in the X-rays and in the radio band the SF is most often defined in terms of flux differences, although there are exceptions, e.g., Fiore et al. (1998) introduced X-ray magnitudes and their differences.

For an analogy with the optical, we used the logarithm of the flux instead of the flux itself, and defined the SF with the following formula:

$S F(\tau) \equiv \sqrt{\frac{\pi}{2}\left\langle\left|\log f_{\mathrm{X}}(t+\tau)-\log f_{\mathrm{X}}(t)\right|\right\rangle^{2}-\sigma_{\mathrm{n}}^{2}}$.

Here, the average of the absolute value of the difference is used, as in di Clemente et al. (1996); $\sigma_{\mathrm{n}}$ is the contribution of the 


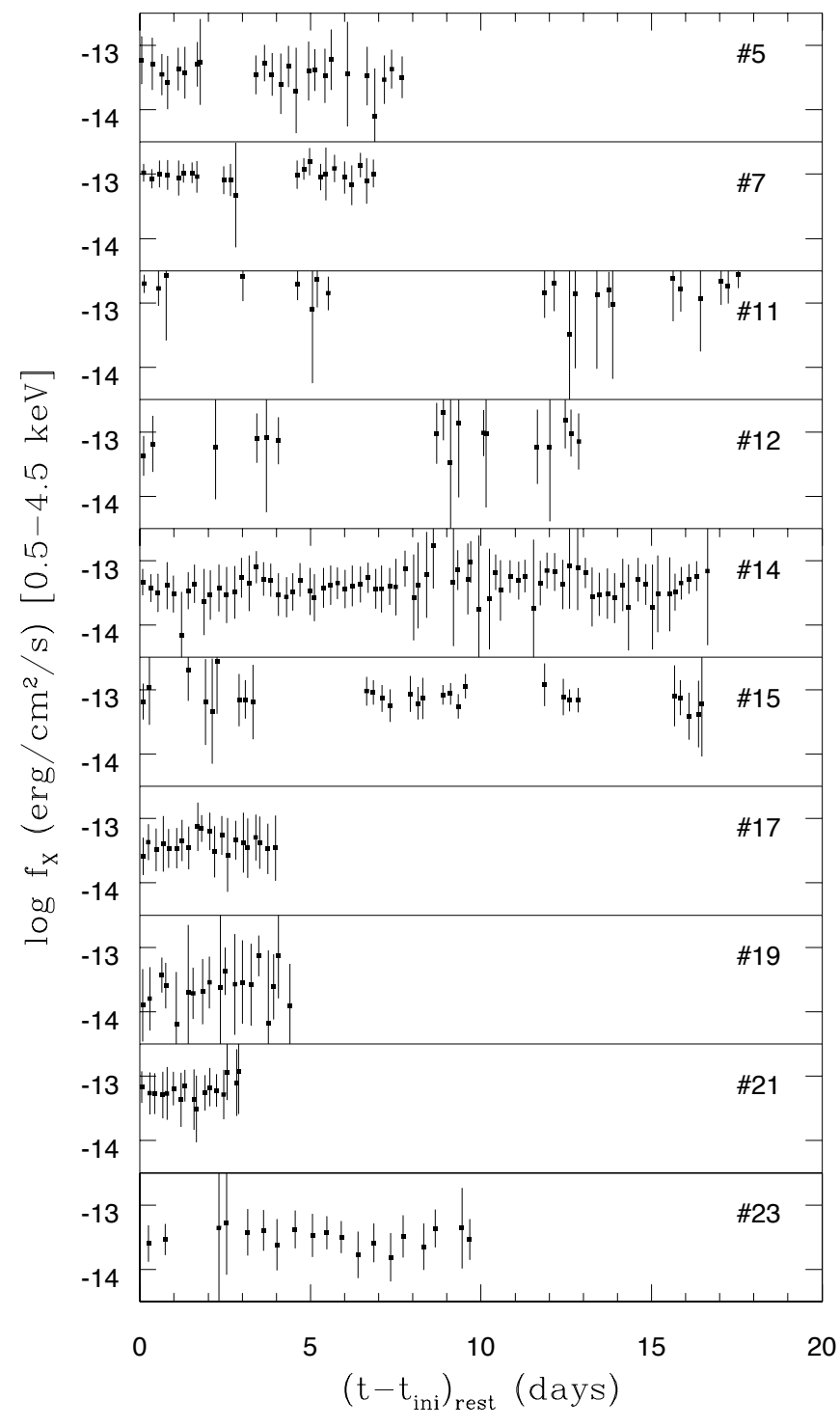

Fig. 3. Some of the best-sampled light curves from the Swift sample. Times are counted from the initial epoch of each light curve, in the restframe. Errors proportional to the inverse square root of the photon count at each epoch are displayed as 3- $\sigma$ values. Errors are discussed in more detail in Sect. 3. Source numbers from Col. 1 of Table 2 are indicated.

photometric noise to the observed variations. $f_{\mathrm{X}}(t)$ and $f_{\mathrm{X}}(t+\tau)$ are two measures of the flux $f_{\mathrm{X}}$ in a given X-ray band at two epochs differing by the lag $\tau$. The factor $\pi / 2$ normalises SF to the rms value in the case of a Gaussian distribution. The X-ray band adopted in this paper is $0.5-4.5 \mathrm{keV}$, and the lag $\tau$ is computed in the rest frame:

$\tau_{\text {rest }}=\tau_{\text {obs }} /(1+z)$.

While a definition in terms of flux differences could also be used for studies of individual sources, our definition with logarithmic differences, Eq. (1), is certainly preferable for an ensemble analysis, otherwise the contribution of faint sources would be negligible compared to that of brighter ones.

We now computed and compared the structure functions for the two samples. While the XMM-Newton sample is much larger than the Swift sample (412 sources vs. 27 sources), the number of epochs is very small for most XMM-Newton sources

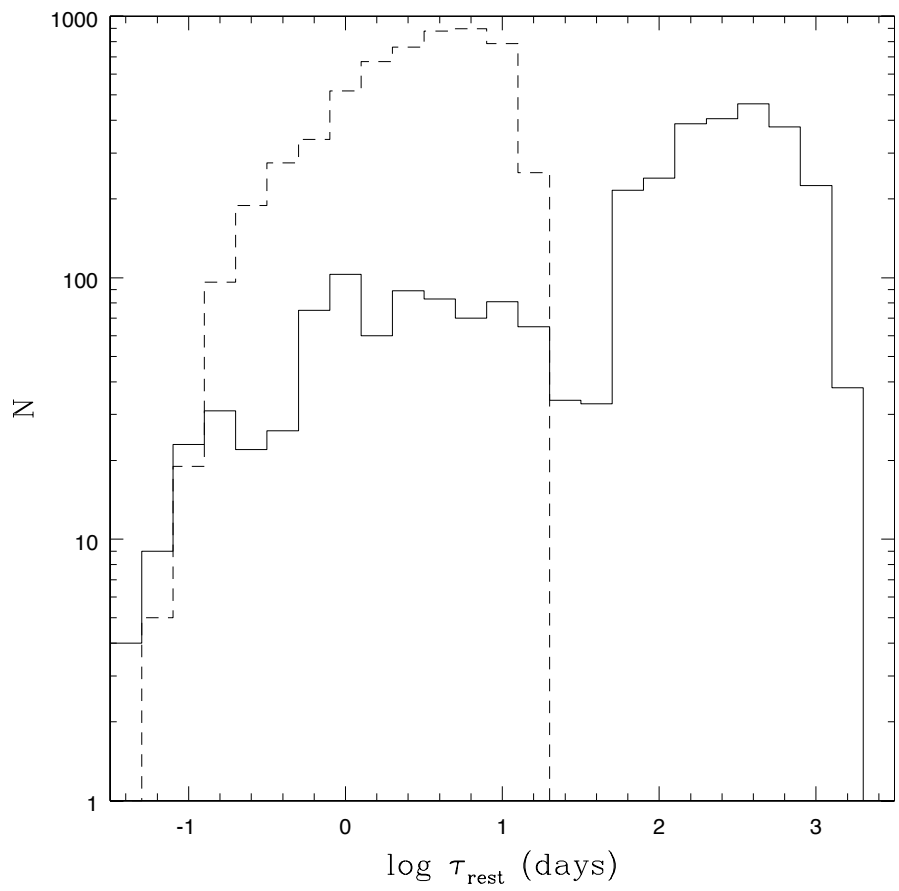

Fig. 4. Histogram of the rest-frame time lags contributing to the structure functions. Continuous histogram: XMM-Newton sample; dashed histogram: Swift sample.

(338/412 $=82 \%$ of the sources having less than 5 epochs), while the Swift light curves (with the adopted binning, see Sect. 2.2) are better sampled, $21 / 27=78 \%$ of the sources having 10 or more bins (or "epochs"), with a mean number $\sim 16$. So the contributions of the two samples to the respective SFs are comparable in number, although different in the time scales sampled. The light-curve of the $k$ th source, with $N_{k}$ epochs, contributes $N_{k}\left(N_{k}-1\right) / 2$ points to $S F\left(\tau_{\text {rest }}\right)$, for all the time lags $\tau_{\text {rest }, i j}=\left|t_{i}-t_{j}\right| /(1+z)$, where $t_{i}$ and $t_{j}$ are two epochs in the observer frame.

This can be seen in Fig. 4, where the histograms of the restframe time lags are shown for the two samples, with bins of $\Delta \log \tau=0.2$ : hundreds of points contribute the most populated bins of each sample, which are days-weeks for the Swift sample and months-years for the XMM-Newton sample. The latter contributes also non-negligibly in the days-weeks range, with several tens of points.

In Figs. 5 and 6 we show the structure functions computed with Eq. (1) for the XMM-Newton and Swift samples, respectively.

To estimate the photometric noise $\sigma_{\mathrm{n}}$ in Eq. (1), we evaluated its contribution in each bin with the following considerations. The quadratic contribution of the noise to the SF is

$$
\begin{aligned}
\sigma_{\mathrm{n}}^{2} & =2\left\langle\left(\delta \log f_{\mathrm{X}}\right)^{2}\right\rangle \approx 2(\log e)^{2}\left\langle\left(\frac{\delta f_{\mathrm{X}}}{f_{\mathrm{X}}}\right)^{2}\right\rangle \\
& =2 \cdot 0.434^{2}\left\langle\frac{1}{N}\right\rangle=0.377 \frac{\sum \frac{1}{N_{k}}}{N_{\mathrm{p}}}
\end{aligned}
$$

where $\delta f_{\mathrm{X}}$ are the flux variations caused by noise alone (excluding source variability), and we assume $\delta f_{\mathrm{X}} / f_{\mathrm{X}}=1 / \sqrt{N}, N$ being the number of counted photons at a given epoch, and its reciprocal is mediated in any given bin of the SF among the $N_{\mathrm{p}}$ points, which are contributed by the various light-curves; $N_{k}$ is 


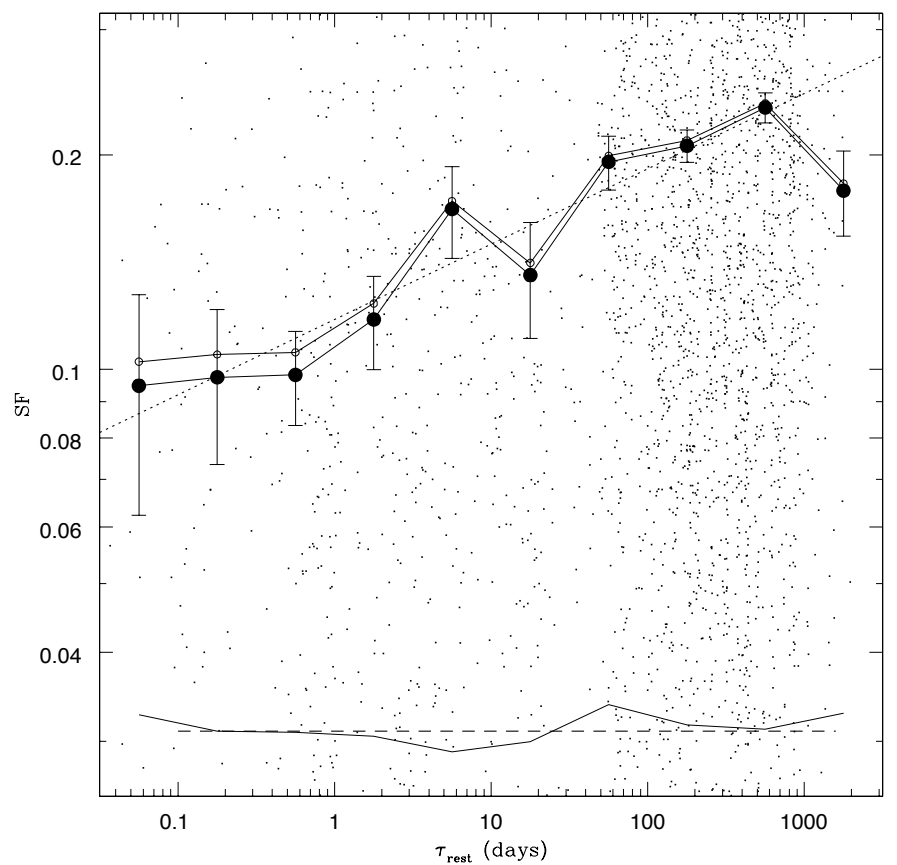

Fig. 5. Structure function for the XMM-Newton sample in bins of $\Delta \log \tau=0.5$. The small empty circles and the continuous line connecting them show the uncorrected SF (i.e., neglecting $\sigma_{\mathrm{n}}$ in Eq. (1)). The larger filled circles and the line connecting them, show the SF corrected for the noise. The continuous line without data points indicates the average value of the noise in each bin, and the dashed, horizontal line is its weighted average, according to the number of points in each bin, adopted in Eq. (1). The dotted line is a weighted least-squares fit to the data of the bins. The small dots are the contributions from pairs of individual measurements at times differing by $\tau$.

the average photon count per epoch of the $k$ th light-curve; the factor 2 is due to the contribution of 2 independent measurements to each flux variation. The values obtained in each bin are connected and shown in Figs. 5 and 6 as thin, continuous lines, while their average values, weighted with the numbers of points in each bin, are shown as dashed lines.

The average values $\sigma_{\mathrm{n}}=0.031$ (XMM-Newton sample) and $\sigma_{\mathrm{n}}=0.163$ (Swift sample) were then inserted in Eq. (1) to compute the SF, which is shown in Figs. 5 and 6, both with and without noise subtraction. The noise so estimated is almost negligible for the XMM-Newton sample, and quite high for the Swift sample. This is mainly because of the smaller effective area of Swift, and also because of the longer exposures of the XMM-Newton observations, which are typically several tens of ks per epoch, while for Swift the light-curves are binned in intervals of $50 \mathrm{ks}$, with effective exposures within a small fraction of the bin, around $10 \mathrm{ks}$.

Although the two SFs appear different before noise subtraction, their slopes and amplitudes agree quite well after correction. We stress that noise subtraction is not parametrical, but consistently derived by the photon counts. The fits shown in Figs. 5 and 6 are least squares of the bin representative points, weighted with the number of individual points in each bin, $\log S F=a+b \log \tau_{\text {rest }}$, or

$S F \propto \tau_{\text {rest }}^{b}$

with consistent slopes, $b=0.10 \pm 0.01$ for the XMM-Newton sample, and $b=0.07 \pm 0.04$ for the Swift sample.

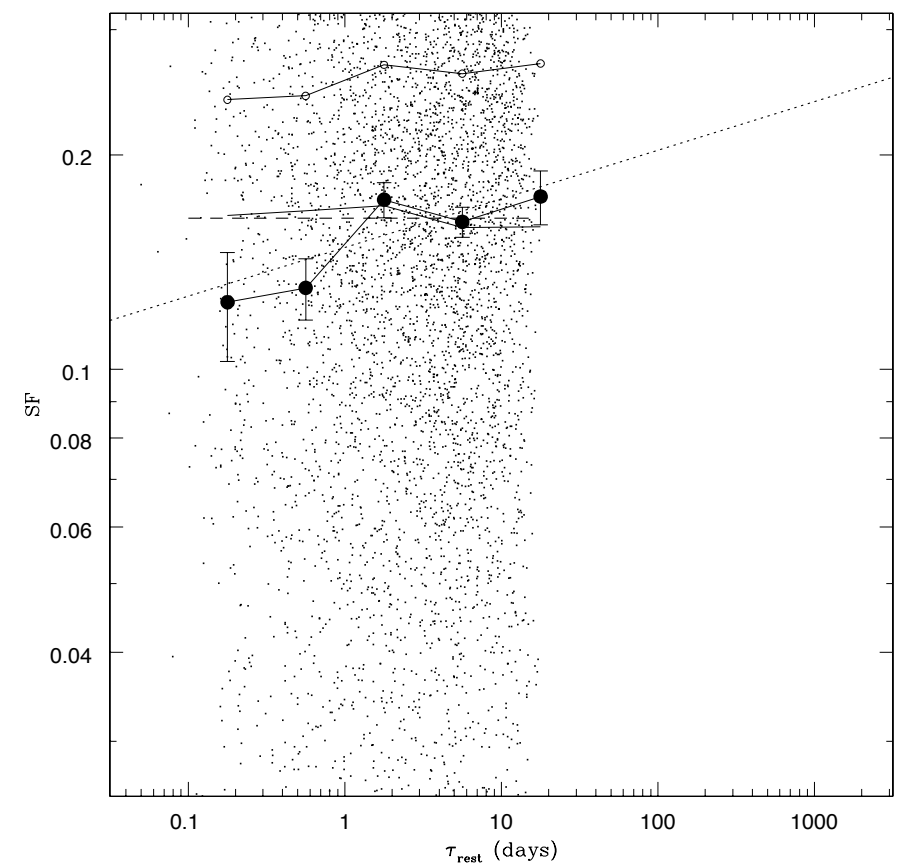

Fig. 6. Structure function for the Swift sample, in bins of $\Delta \log \tau=0.5$. The small empty circles and the continuous line connecting them show the uncorrected SF (i.e., neglecting $\sigma_{\mathrm{n}}$ in Eq. (1)). The larger, filled circles and the line connecting them show the SF corrected for the noise. The continuous line without data points indicates the average value of the noise in each bin, and the dashed, horizontal line is their weighted average, according to the number of points in each bin, adopted in Eq. (1). The dotted line is a weighted least-squares fit to the data of the bins. The small dots are the contributions from pairs of individual measurements at times differing by $\tau$.

\subsection{Relation with the PSD}

X-ray variability of individual sources is usually analysed in terms of the PSD. This has been often described by a powerlaw, $P(f) \propto f^{-\alpha}, \alpha \sim 1.5$, (e.g. Lawrence \& Papadakis 1993). However, one or two breaks in the PSD of nearby AGNs have also been detected (e.g. Markowitz et al. 2003; O'Neill et al. 2005), and the PSD has been found to have a power-law exponent $\alpha \approx 2$ for $f>f_{\mathrm{HFB}}, \alpha \approx 1$ for $f_{\mathrm{LFB}}<f<f_{\mathrm{HFB}}$, and in some cases $\alpha \approx 0$ for $f<f_{\mathrm{LFB}}$. In turn, the high-frequency break has been found to be related to the mass of the central $\mathrm{BH}$ (e.g. Papadakis 2004).

An SF with the form of a single power-law as in Eq. (4) is equivalent to a single power-law PSD if the frequency range extends from 0 to $\infty$. Then a simple relation between the exponents holds (e.g. Kawaguchi et al. 1998; Bauer et al. 2009; Emmanoulopoulos et al. 2010):

$\alpha=1+2 b$.

The slope of our SF, $b \lesssim 0.1$, would then correspond to a PSD exponent $\alpha \lesssim 1.2$, slightly flatter than the reference value $\alpha \sim$ 1.5 (Lawrence \& Papadakis 1993).

However, Eq. (5) does not straightforwardly apply when the PSD contains a break. Emmanoulopoulos et al. (2010) produced 2000 artificial light-curves with a PSD shaped as a broken power-law with a break at a given value $f_{\mathrm{B}}$, and estimate the corresponding SFs. Figures 10 and 11 of Emmanoulopoulos et al. (2010) show that SFs also display a break whose distribution peaks around $\tau_{\mathrm{B}} \sim 1 / f_{\mathrm{B}}$, but the SF slopes before and after 


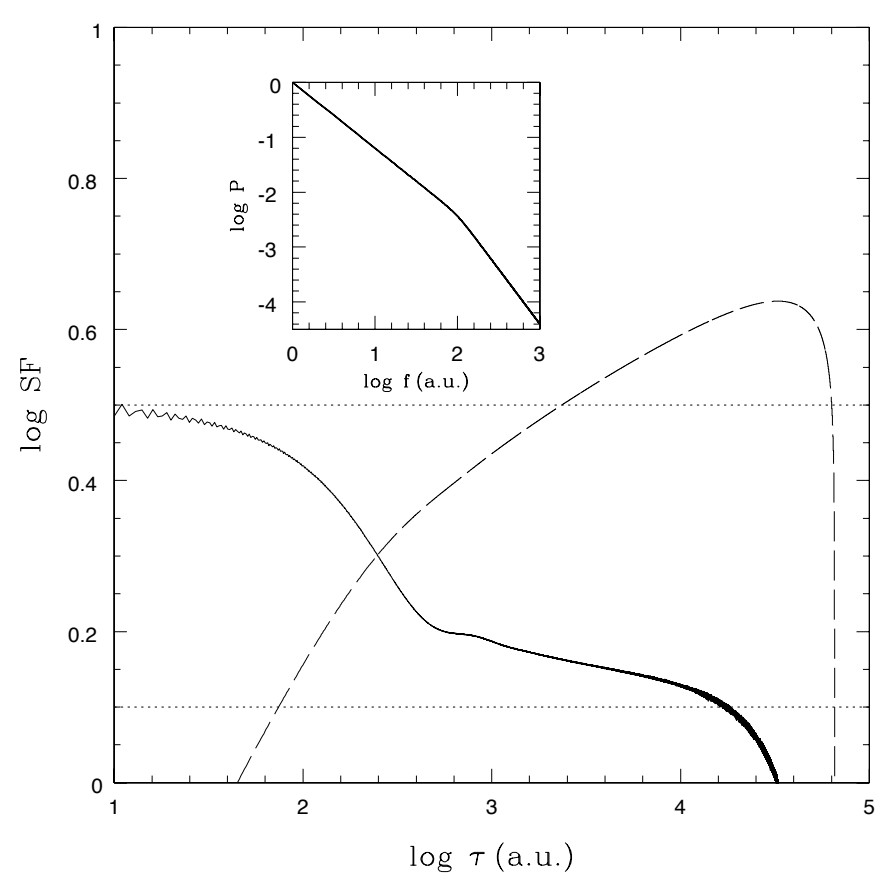

Fig. 7. SF (dashed line) and its slope (continuous line) computed from a PSD shaped as a broken power-law, with $\alpha_{1}=1.2$ and $\alpha_{2}=2$, shown in the inset. The dotted lines indicate the values of the power-law exponent $b$, expected from Eq. (5) for the single power-law case, $b_{1}=0.1$, $b_{2}=0.5$. The cut-off in the SF at long $\tau$ is caused by the finite number of Fourier frequencies used in the FFT calculation. Time lags and Fourier frequencies are in arbitrary units.

this break do not agree with the relation of Eq. (5). In particular, the SF appears flatter than Eq. (5) below the break, and steeper above the break, resulting in less bending.

To analyse the relation between the shapes of PSD and SF, we evaluated the SF numerically via fast Fourier transform (FFT) techniques according to the relation $S F(\tau)=2 \int_{0}^{\infty}(1-$ $\cos (2 \pi f \tau) P(f) \mathrm{d} f$ (e.g. Emmanoulopoulos et al. 2010), for a PSD shaped as a broken power-law. The result, shown in Fig. 7 for input PSD spectral indexes $\alpha_{1}=1.2, \alpha_{2}=2$, is an SF shaped approximately as a broken power-law, but with a slope changing gradually and with less bending, which confirms the result by Emmanoulopoulos et al. (2010).

The above results suggest that we should expect some evidence of a break in the SF of AGNs with a typical broken powerlaw PSD.

\subsection{Dependence on mass and bolometric luminosity}

McHardy et al. (2006) proposed that the high-frequency break is related not only to the black hole mass, $M_{\mathrm{BH}}$, but also to the accretion rate in units of its Eddington value, $\dot{m}_{\mathrm{E}} \approx L_{\mathrm{bol}} / L_{\mathrm{Edd}}$, and found the following relation

$\log \tau_{\text {break }}($ days $)=2.1 \log M_{6}-0.98 \log L_{44}-2.32$,

where we abbreviate $M_{6}=M_{\mathrm{BH}} / 10^{6} M_{\odot}$ and $L_{44}=$ $L_{\text {bol }} / 10^{44} \mathrm{erg} / \mathrm{s}$.

However, while for the light curves of individual objects the relation between SF and PSD is relatively simple, for an ensemble SF the different positions of the breaks should combine in the ensemble SF, possibly smoothing the result, depending on how the variability amplitude changes with $M_{\mathrm{BH}}$ and/or $L_{\mathrm{bol}}$.

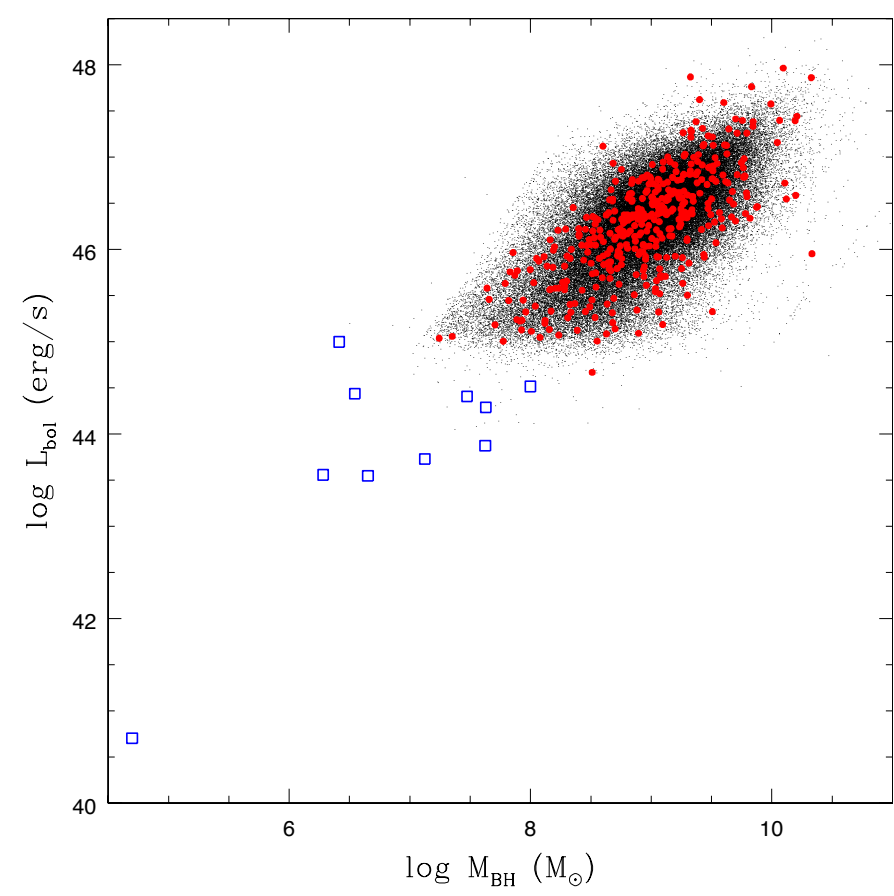

Fig. 8. Black hole masses and bolometric luminosities of AGN samples. Black dots: $\sim 100000$ sources from the Shen et al. (2011) catalogue. Red filled circles: XMM-Newton sample. Blue empty squares: low-luminosity AGNs from Uttley \& McHardy (2005).

To find any break in the SF, we segregated our XMM-Newton sample according to $M_{\mathrm{BH}}$ and $L_{\mathrm{bol}}$ values. Estimates of the masses and bolometric luminosities were extracted from the catalogue of quasar properties by Shen et al. (2011). We show in Fig. 8 the distribution of $\sim 100000$ AGNs from that catalogue in the plane $M_{\mathrm{BH}}-L_{\mathrm{bol}}$, as well as the same distribution for our XMM-Newton sample, which appears quite similar, despite its smaller population (412 sources). We also show in the same figure some low-luminosity AGNs from Uttley \& McHardy (2005), which will be discussed below.

We then plot in Fig. 9 the structure function for $X M M$-Newton subsamples binned in intervals of $\log M_{\mathrm{BH}}$ and $\log L_{\text {bol }}$, with bin width $\Delta \log M_{\mathrm{BH}}=\Delta \log L_{\mathrm{bol}}=0.5$. The $\mathrm{SF}$ is shown for subsamples with at least $30 \mathrm{SF}$ points, in the range of masses $10^{7.5} M_{\odot}<M_{\mathrm{BH}}<10^{10} M_{\odot}$ and luminosities $10^{45} \mathrm{erg} / \mathrm{s}<L_{\text {bol }}<10^{47.5} \mathrm{erg} / \mathrm{s}$. The total number of SF points is reported in each box, as well as the average SF slope (weighted with the number of points in each bin of $\tau_{\text {rest }}$ ), and the expected value of $\log \tau_{\text {break }}$, according to Eq. (6). The SF of the total XMM-Newton sample is also reported for comparison.

Our results do not support the existence of a break in the SF, expected following Eq. (6). However, we note that the analysis by McHardy et al. (2006) is based on a few AGNs with quite low luminosities and masses (see Uttley \& McHardy 2005), compared to our XMM-Newton sample, and the Shen et al. (2011) catalogue, see Fig. 8.

The absence of a break in our results could be understood if Eq. (6), which appears to hold for AGNs with $M_{\mathrm{BH}} \lesssim 10^{8} M_{\odot}$ and $L_{\mathrm{bol}} \lesssim 10^{45} \mathrm{erg} / \mathrm{s}$, would not apply for larger masses and higher luminosities.

McHardy et al. (2006) associated the break time scale to a thermal or viscous time scale related to the inner radius of the accretion disk, and identify this with the transition radius $R_{\mathrm{tr}}$ predicted by Liu et al. (1999), based on evaporation of the inner disk in low Eddington ratio AGNs, describing the transition 


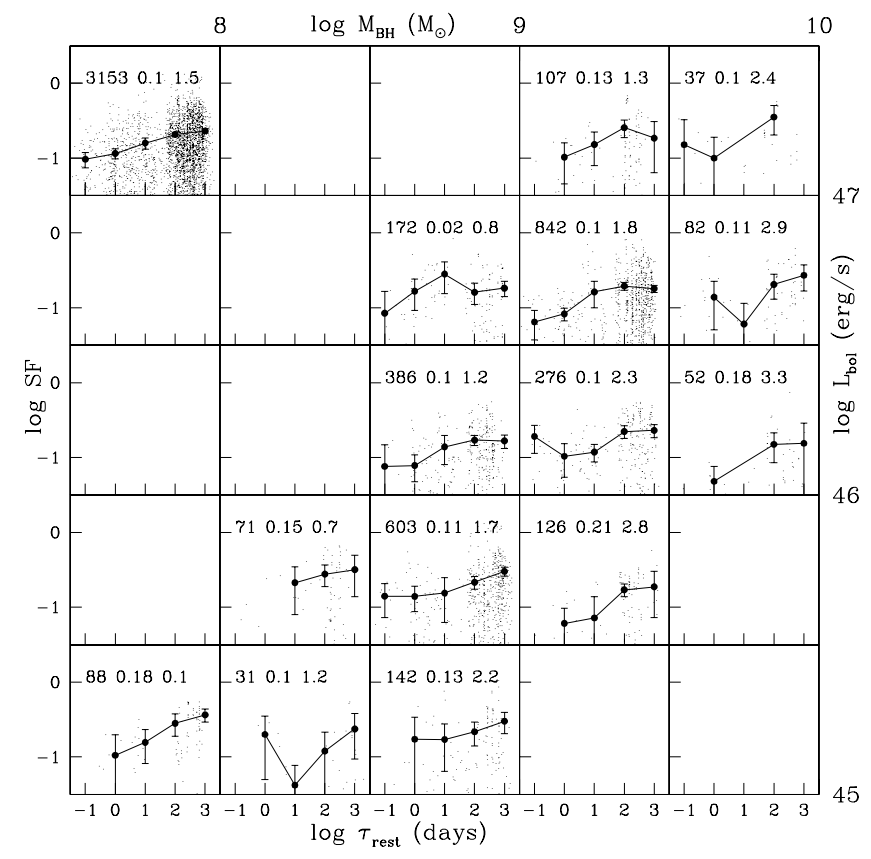

Fig. 9. Structure function for XMM-Newton subsamples binned in intervals of $\log M_{\mathrm{BH}}$ and $\log L_{\mathrm{bol}}$, with bin width $\Delta \log M_{\mathrm{BH}}=\Delta \log L_{\mathrm{bol}}=$ 0.5 . Values of $M_{\mathrm{BH}}$ and $L_{\mathrm{bol}}$ are reported in the upper and right axes, respectively. Subsamples with less than $30 \mathrm{SF}$ points are not shown. The $\mathrm{SF}$ of the total XMM-Newton sample is reported, for comparison, in the first box in the upper left corner. The values reported in each box are the number of SF points, the average SF slope, and the expected value of $\log \tau_{\text {break }}$, according to Eq. (6). Contributions from pairs of individual measurements are also shown (dots).

between an external cool thin disk and an inner, hot, advectiondominated accretion flow (ADAF). This model clearly does not apply to high-luminosity QSOs (see, e.g. Narayan et al. 1998).

\subsection{Dependence on X-ray luminosity and redshift}

Many authors have found inverse dependences of the X-ray variability on the X-ray luminosity $L_{\mathrm{X}}$. Different variability indexes are used, so they must be briefly recalled to make comparisons.

Most authors use the normalised excess variance (e.g. Nandra et al. 1997; Vaughan et al. 2003), defined as $\sigma_{\mathrm{NXS}}^{2}=$ $\left(S^{2}-\sigma_{\mathrm{n}}^{2}\right) / \bar{x}^{2}$, where $S^{2}$ is the total variance of the light curve, $\sigma_{\mathrm{n}}^{2}$ is the mean square error, and $\bar{x}$ is the mean of $N$ total measurements; or the square root of it, which is also referred to as fractional variability amplitude, $F_{\text {var }}$ (e.g. Markowitz \& Edelson 2004).

Green et al. (1993) used the normalised variability amplitude, square root of the power at a specific frequency, normalised to the mean count rate of the related light curve. Lawrence \& Papadakis (1993) used the amplitude of the power spectrum at a specific frequency.

As pointed out by Lawrence \& Papadakis (1993), $\sigma_{\mathrm{NXS}}^{2}$ and $F_{\text {var }}$ depend on the length of the monitored time interval. Moreover, we notice that they depend on redshift, because the time interval must be properly measured in the rest-frame of the source, as stressed by Giallongo et al. (1991) for the optical variability, and by Papadakis et al. (2008) for the X-ray case. So the comparison between different results must be taken with some caution. With these limitations in mind, and calling $I_{\mathrm{var}}$ a generic variability index (or its square root where appropriate), most of the previous results on the variability dependence on luminosity can be expressed in power-law form, $I_{\mathrm{var}} \propto L_{\mathrm{X}}^{-k}$. Values for the exponent $k$ are usually about $\sim 0.3$, for time scales of days, and for samples including Seyfert galaxies and/or low-z QSOs (Green et al. 1993; Lawrence \& Papadakis 1993; Nandra et al. 1997; Markowitz \& Edelson 2004). Similar values are found also for higher redshift QSOs, e.g. by Manners et al. (2002), up to $z=2(k=0.27$, still for time scales of days), and by Papadakis et al. (2008), up to $z \sim 3.4$ ( $k=0.33$, for time scales of tens of days). Stronger dependences are instead found by Paolillo et al. (2004) $(k \sim 0.65$, in the redshift range $0.5<z<1.3)$ and by Almaini et al. (2000) $(k=0.75$, for $z<0.5)$. For longer time scales (years), a few analyses have been performed, e.g., Markowitz \& Edelson (2004) found a weaker dependence, $k \sim 0.13$.

With the analysis of the rest-frame structure function, we can properly compare variability amplitudes at various time lags, and provide an unbiased characterisation of the dependence of variability on luminosity and redshift. In Fig. 10 we show the SFs for four luminosity bins between $L_{\mathrm{X}}=10^{43.5} \mathrm{erg} / \mathrm{s}$ and $L_{\mathrm{X}}=10^{45.5} \mathrm{erg} / \mathrm{s}$ : a clear and strong dependence on $L_{\mathrm{X}}$ appears. A change in the slope of the SF is also present (between $\sim 0.04$ and 0.14), implying that a different dependence on $L_{X}$ is expected for different time lags. To see this, we re-plot in Fig. 11 the SF data vs $L_{\mathrm{X}}$ for two different bins of time lag, centred on 1 day and 100 days, respectively. The least-squares fits, weighted with the number of measurements in each bin, correspond to power-law exponent $k=0.42 \pm 0.03$ for the shorter time scale, a slightly stronger dependence, compared to the results by most previous authors. For the longer time scale (100 days), our result is $k=0.21 \pm 0.07$, which approximately agrees with the trend found by Markowitz \& Edelson (2004).

A simple interpretation of the decrease of variability with luminosity $(L)$ is the superposition of $N$ randomly flaring subunits. This was already considered in early studies of optical variability (e.g. Pica \& Smith 1983; Aretxaga et al. 1997), and, in its simplest version of independent and identical flares, would predict a variability amplitude $\propto N^{-1 / 2} \propto L^{-1 / 2}$. In the X-ray domain, several authors have also considered the same argument (Green et al. 1993; Nandra et al. 1997; Almaini et al. 2000; Manners et al. 2002). The observed shallower slope can be understood invoking a correlation among flares (e.g. Green et al. 1993), or a dependence of the amplitude of the flares on the luminosity of the source (Almaini et al. 2000). We stress that a simple scaling of the flare amplitude with the luminosity of the source cannot account for the change in the slope of the SF with luminosity, shown in Fig. 10, unless some correlation among the flares is also introduced.

Instead of multiple flaring subunits, models based on the variability of a single region have also been considered, e.g., Almaini et al. (2000) explained the dependence of variability on luminosity, invoking a relation between the luminosity and the size of the varying region, which produces a shift of the PSD in the frequency direction, with unchanged slope, under the assumption of self-similar scaling of the variable region. However, a PSD with slope independent on luminosity is inconsistent with our results on the SF (see Fig. 10), implying that a deviation from self-similarity should be considered.

The stronger dependences on $L_{X}$ found by Almaini et al. (2000) and Paolillo et al. (2004) are accompanied by the suggestion of a possible increase of the variability with redshift. Almaini et al. (2000) find an opposite dependence on luminosity $(k=-0.3)$ for sources at $z>0.5$, which could be caused by an increase with $z$. Paolillo et al. (2004) measure a higher 


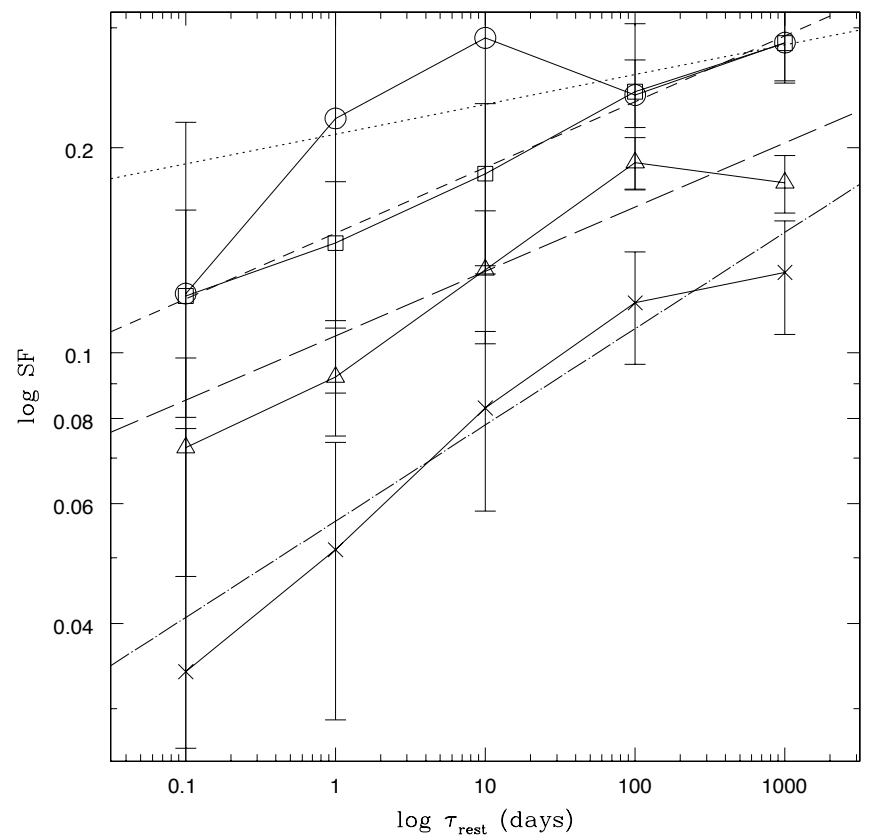

Fig. 10. Structure function in bins of X-ray luminosity, represented as points connected by continuous lines. Straight lines with different dash styles: least-squares fits weighted according to the number of points in each bin of time lag. $10^{43.5} \mathrm{erg} / \mathrm{s}<L_{X}<10^{44} \mathrm{erg} / \mathrm{s}$ : circles, dotted lines; $10^{44} \mathrm{erg} / \mathrm{s}<L_{\mathrm{X}}<10^{44.5} \mathrm{erg} / \mathrm{s}$ : squares, short-dashed lines; $10^{44.5} \mathrm{erg} / \mathrm{s}<L_{\mathrm{X}}<10^{45} \mathrm{erg} / \mathrm{s}$ : triangles, long-dashed lines; $10^{45} \mathrm{erg} / \mathrm{s}<L_{\mathrm{X}}<10^{45.5} \mathrm{erg} / \mathrm{s}$ : crosses, dot-dashed lines.

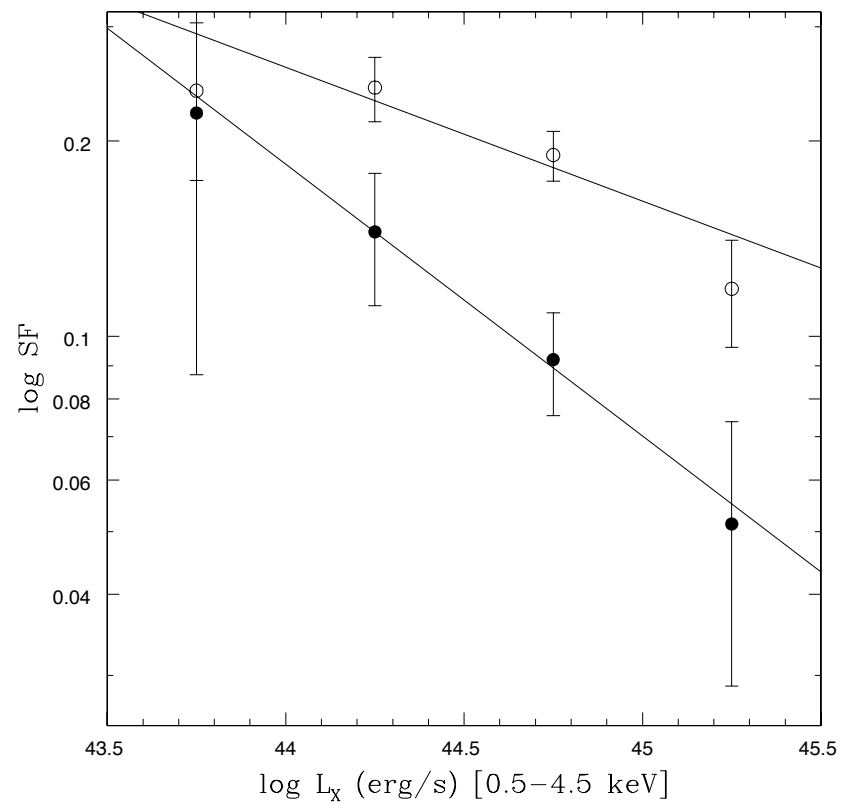

Fig. 11. Dependence of the SF on $L_{\mathrm{X}}$. Filled circles: $\tau_{\text {rest }}=1 \mathrm{~d}$; open circles: $\tau_{\text {rest }}=100 \mathrm{~d}$. Lines: weighted least-squares fits, according to the number of points in each bin of $L_{\mathrm{X}}$.

variability for sources at $z>1.3$ than for their low- $z$ counterparts of similar luminosity. Manners et al. (2002) also reported tentative evidence of a stronger variability for sources at $z>2$. Finally, Papadakis et al. (2008) compared the variability of highredshift AGNs in the Lockman Hole region with that of nearby AGNs by Markowitz \& Edelson (2004), finding evidence of an increase with redshift.

Owing to the strong correlation of sources in the $L_{X}-z$ plane (Fig. 1), we limited our analysis of the $z$-dependence to the

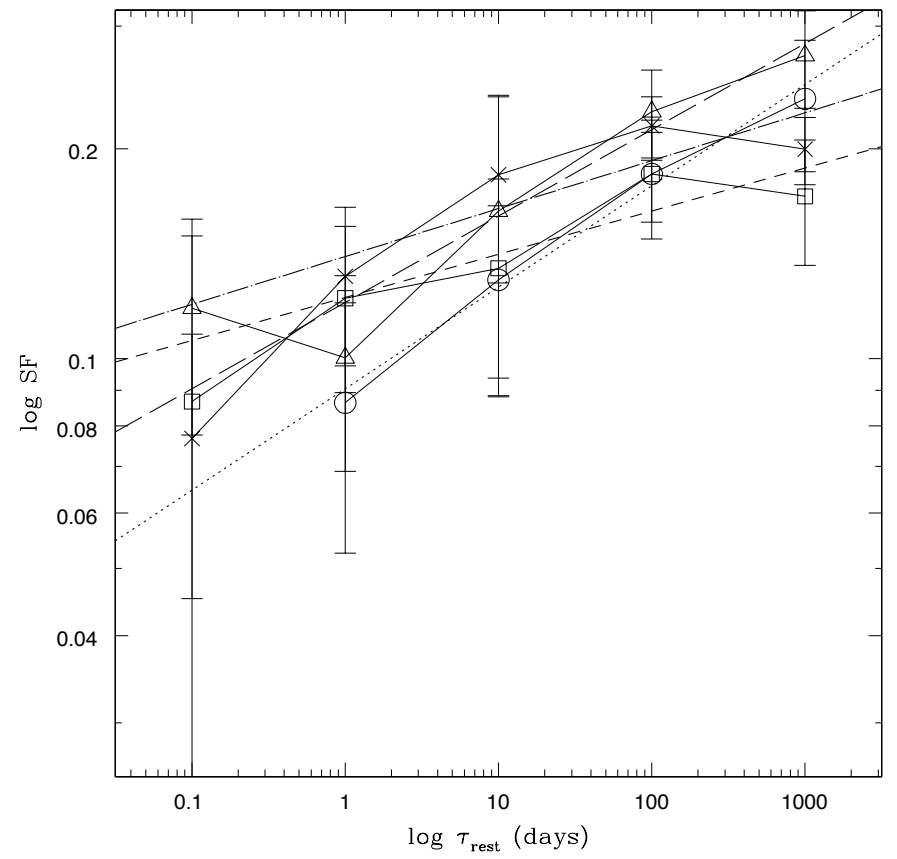

Fig. 12. Structure function in bins of redshift for sources in the luminosity interval $10^{44} \mathrm{erg} / \mathrm{s}<L_{X}<10^{45} \mathrm{erg} / \mathrm{s}$, represented as points connected by continuous lines. Straight lines with different dash styles: least-squares fits, weighted according to the number of points in each bin of time lag. $0<z \leq 1$ : circles, dotted lines; $1<z \leq 1.4$ : squares, short-dashed lines; $1.4<z \leq 1.8$ : triangles, long-dashed lines; $1.8<z \leq 4.5$ : crosses, dot-dashed lines.

sources in the luminosity interval $10^{44} \mathrm{erg} / \mathrm{s}<L_{\mathrm{X}}<10^{45} \mathrm{erg} / \mathrm{s}$, and divided the sample into four equally populated redshift bins, $0<z \leq 1,1<z \leq 1.4,1.4<z \leq 1.8,1.8<z \lesssim 4.5$. The result, displayed in Fig. 12, suggests the presence of a weak trend with redshift at intermediate time scales, while at short and long timescales the behaviour appears unclear and possibly nonmonotonic.

To investigate this dependence in more detail, we computed partial correlation coefficients of variability with redshift, considering all individual variations that contribute to the SF. While the ordinary correlation coefficient indicates no correlation, $r_{V z}=-0.06$, with probability $P(>r)=0.001$, the first-order partial correlation coefficient, which takes account of the dependence on $L_{X}$, is

$r_{V z, L}=\left(r_{V z}-r_{V L} r_{z L}\right) / \sqrt{\left(1-r_{V L}^{2}\right)\left(1-r_{z L}^{2}\right)}=0.125$,

with probability $P(>r)=10^{-12}$, suggesting the presence of a weak, intrinsic correlation. We also calculated the second-order partial correlation coefficient (Kendall \& Stuart 1977), which compensates for both the dependences on $L_{X}$ and on the time lag $\tau$, and still strengthens the correlation:

$r_{V z, L \tau}=\left(r_{V z, L}-r_{V \tau, L} r_{z \tau, L}\right) / \sqrt{\left(1-r_{V \tau, L}^{2}\right)\left(1-r_{z \tau, L}^{2}\right)}=0.127$.

The probability is in this case $P(>r)=6 \times 10^{-13}$.

\section{Discussion}

The analysis of X-ray variability of AGNs has previously been performed mainly for individual nearby Seyferts or for small samples of them, and only a few works extend the study to large 
samples in wide ranges of luminosity and redshift (e.g. Almaini et al. 2000; Manners et al. 2002; Paolillo et al. 2004). Our study presents the first ensemble analysis based on the structure function. This is to be preferred for statistic studies compared with a PSD analysis, because SF operates in the time domain, is less dependent on irregular sampling, and allows for an analysis even with very few epochs. The SF is also preferable compared with the analysis of fractional variability and excess variance, because these parameters are biased by the duration of the monitoring time interval in the rest-frame, and thus on cosmological time dilation.

Our variability analysis, based on two different serendipitously selected samples extracted from the catalogues of XMM-Newton (Watson et al. 2009) and Swift (Puccetti et al. 2011), gives statistically consistent results in the two cases, with the SF described by a power law of the time lag, with exponent $b=0.10 \pm 0.01$ (XMM-Newton $)$ or $b=0.07 \pm 0.04$ (Swift). This would correspond to a PSD with power law exponent $\alpha \approx 1.2$ for the case of a single-power-law PSD, which is within the range of exponents found for nearby Seyferts (Lawrence \& Papadakis 1993).

While the PSD of local low-luminosity AGNs often shows one or two breaks, we do not find evidence of breaks in the SF, even dividing the analysis in bins of $M_{\mathrm{BH}}$ and $L_{\mathrm{bol}}$. However, while a break at a time lag roughly proportional to the black hole mass is expected for local AGNs, our results do not support this expectation for more luminous AGNs and QSOs. This suggests that the relation found by McHardy et al. (2006), reported in Eq. (6), cannot be extrapolated to high bolometric luminosities and large black hole masses, possibly because the transition between an external cool thin disk and an inner ADAF (Liu et al. 1999) does not apply in the high-Eddington ratio regime.

We confirm a strong anti-correlation of the variability with $\mathrm{X}$-ray luminosity, as $L_{\mathrm{X}}^{-0.42}$ and as $L_{\mathrm{X}}^{-0.21}$ for time lags $\sim 1$ day and $\sim 100$ days, respectively. This approximately agrees with most previous authors (Green et al. 1993; Lawrence \& Papadakis 1993; Nandra et al. 1997; Markowitz \& Edelson 2004; Papadakis et al. 2008).

The behaviour of the slope and amplitude of the SF as a function of the luminosity implies that (i) for a model of multiple flaring subunits, they cannot be uncorrelated; (ii) for a model with a single varying region self-similar scaling with luminosity cannot hold.

We find evidence in support of a weak, intrinsic, increase of the average X-ray variability with redshift. The dependence, however, appears tangled with that on the time lag. This suggests that different processes could dominate the variability at short and long time scales, and that their relative importance changes with the redshift.

Acknowledgements. We are grateful to Paolo Giommi, Maurizio Paolillo, Matteo Perri, and Simonetta Puccetti for useful discussions. S.T. acknowledges financial support through Grant ASI I/088/06/0. Part of this work is based on archival data, software or on-line services provided by the ASI Science Data Center (ASDC). This research made use of the XMM-Newton Serendipitous Source Catalogue, which is a collaborative project involving the whole Science Survey Center Consortium. Funding for the SDSS and SDSS-II was provided by the Alfred P. Sloan Foundation, the Participating Institutions, the National Science Foundation, the US Department of Energy, the National Aeronautics and Space Administration, the Japanese Monbukagakusho, the Max Planck Society, and the Higher Education Funding Council for England. The SDSS was managed by the Astrophysical Research Consortium for the Participating Institutions.

\section{References}

Abramowicz, M. A., Bao, G., Lanza, A., \& Zhang, X. 1991, A\&A, 245, 454 Abrassart, A., \& Czerny, B. 2000, A\&A, 356, 475

Almaini, O., Lawrence, A., Shanks, T., et al. 2000, MNRAS, 315, 325

Aretxaga, I., Cid Fernandes, R., \& Terlevich, R. J. 1997, MNRAS, 286, 271

Arévalo, P., Uttley, P., Lira, P., et al. 2009, MNRAS, 397, 2004

Barr, P., \& Mushotzky, R. F. 1986, Nature, 320, 421

Bauer, A., Baltay, C., Coppi, P., et al. 2009, ApJ, 696, 1241

Brinkmann, W., Sembay, S., Griffiths, R. G., et al. 2001, A\&A, 365, L162

Chevallier, L., Collin, S., Dumont, A., et al. 2006, A\&A, 449, 493

Collier, S., \& Peterson, B. M. 2001, ApJ, 555, 775

Collin-Souffrin, S. 1991, A\&A, 249, 344

Cristiani, S., Trentini, S., La Franca, F., et al. 1996, A\&A, 306, 395

Czerny, B., Różańska, A., Dovčiak, M., Karas, V., \& Dumont, A.-M. 2004 A\&A, 420, 1

de Vries, W. H., Becker, R. H., \& White, R. L. 2003, AJ, 126, 1217

di Clemente, A., Giallongo, E., Natali, G., Trevese, D., \& Vagnetti, F. 1996, ApJ, 463, 466

Doroshenko, V. T., Sergeev, S. G., Efimov, Y. S., Klimanov, S. A., \& Nazarov, S. V. 2009, Astron. Lett., 35, 361

Emmanoulopoulos, D., McHardy, I. M., \& Uttley, P. 2010, MNRAS, 404, 931

Favre, P., Courvoisier, T. J.-L., \& Paltani, S. 2005, A\&A, 443, 451

Fiore, F., Laor, A., Elvis, M., Nicastro, F., \& Giallongo, E. 1998, ApJ, 503, 607

Gehrels, N., Chincarini, G., Giommi, P., et al. 2004, ApJ, 611, 1005

Giallongo, E., Trevese, D., \& Vagnetti, F. 1991, ApJ, 377, 345

Gliozzi, M., Brinkmann, W., O’Brien, P. T., et al. 2001, A\&A, 365, L128

Green, A. R., McHardy, I. M., \& Lehto, H. J. 1993, MNRAS, 265, 664

Haardt, F., \& Maraschi, L. 1991, ApJ, 380, L51

Hughes, P. A., Aller, H. D., \& Aller, M. F. 1992, ApJ, 396, 469

Iyomoto, N., \& Makishima, K. 2001, MNRAS, 321, 767

Kawaguchi, T., Mineshige, S., Umemura, M., \& Turner, E. L. 1998, ApJ, 504, 671

Kendall, M., \& Stuart, A. 1977, The advanced theory of statistics. 1: Distribution theory, ed. M. Kendall, \& A. Stuart

Lawrence, A., \& Papadakis, I. 1993, ApJ, 414, L85

Lehto, H. J. 1989, in Two Topics in X-Ray Astronomy, Vol. 1: X Ray Binaries. Vol. 2: AGN and the X Ray Background, ed. J. Hunt, \& B. Battrick, ESA Spec. Publ., 296, 499

Liu, B. F., Yuan, W., Meyer, F., Meyer-Hofmeister, E., \& Xie, G. Z. 1999, ApJ, 527, L17

MacLeod, C. L., Ivezić, Ž., Kochanek, C. S., et al. 2010, ApJ, 721, 1014

Manners, J., Almaini, O., \& Lawrence, A. 2002, MNRAS, 330, 390

Markowitz, A., \& Edelson, R. 2004, ApJ, 617, 939

Markowitz, A., Edelson, R., Vaughan, S., et al. 2003, ApJ, 593, 96

Marshall, K., Ryle, W. T., \& Miller, H. R. 2008, ApJ, 677, 880

McHardy, I. M., Koerding, E., Knigge, C., Uttley, P., \& Fender, R. P. 2006, Nature, 444, 730

Miniutti, G., \& Fabian, A. C. 2004, MNRAS, 349, 1435

Nandra, K., George, I. M., Mushotzky, R. F., Turner, T. J., \& Yaqoob, T. 1997, ApJ, 476, 70

Narayan, R., Mahadevan, R., \& Quataert, E. 1998, in Theory of Black Hole Accretion Disks, ed. M. A. Abramowicz, G. Bjornsson, \& J. E. Pringle, 148

O’Neill, P. M., Nandra, K., Papadakis, I. E., \& Turner, T. J. 2005, MNRAS, 358 , 1405

Paolillo, M., Schreier, E. J., Giacconi, R., Koekemoer, A. M., \& Grogin, N. A. 2004, ApJ, 611, 93

Papadakis, I. E. 2004, MNRAS, 348, 207

Papadakis, I. E., Chatzopoulos, E., Athanasiadis, D., Markowitz, A., \& Georgantopoulos, I. 2008, A\&A, 487, 475

Pica, A. J., \& Smith, A. G. 1983, ApJ, 272, 11

Puccetti, S., Capalbi, M., Giommi, P., et al. 2011, A\&A, 528, A122

Schneider, D. P., Richards, G. T., Hall, P. B., et al. 2010, AJ, 139, 2360

Shemmer, O., Romano, P., Bertram, R., et al. 2001, ApJ, 561, 162

Shen, Y., Richards, G. T., Strauss, M. A., et al. A. 2011, ApJS, 194, 45

Simonetti, J. H., Cordes, J. M., \& Heeschen, D. S. 1985, ApJ, 296, 46

Trevese, D., \& Vagnetti, F. 2002, ApJ, 564, 624

Trevese, D., Kron, R. G., Majewski, S. R., Bershady, M. A., \& Koo, D. C. 1994 ApJ, 433, 494

Trevese, D., Kron, R. G., \& Bunone, A. 2001, ApJ, 551, 103

Uttley, P., \& McHardy, I. M. 2005, MNRAS, 363, 586

Uttley, P., McHardy, I. M., \& Papadakis, I. E. 2002, MNRAS, 332, 231

Vagnetti, F., Turriziani, S., Trevese, D., \& Antonucci, M. 2010, A\&A, 519, A17

Vanden Berk, D. E., Wilhite, B. C., Kron, R. G., et al. 2004, ApJ, 601, 692

Vaughan, S., Edelson, R., Warwick, R. S., \& Uttley, P. 2003, MNRAS, 345, 1271

Watson, M. G., Schröder, A. C., Fyfe, D., et al. 2009, A\&A, 493, 339

Zhang, Y. H., Treves, A., Celotti, A., et al. 2002, ApJ, 572, 762 
Table 1. XMM-Newton sample.

\begin{tabular}{|c|c|c|c|c|c|c|c|}
\hline $\begin{array}{l}N_{\text {sou }} \\
\text { (1) }\end{array}$ & $\begin{array}{c}\text { Name } \\
(2)\end{array}$ & $\begin{array}{c}z \\
(3)\end{array}$ & $\begin{array}{c}N_{\text {epo }} \\
\text { (4) }\end{array}$ & $\underset{(5)}{\log f_{X}}$ & $\begin{array}{c}\log L_{X} \\
\text { (6) }\end{array}$ & $\begin{array}{c}\log \tau_{\text {min }} \\
\text { (7) }\end{array}$ & $\begin{array}{c}\log \tau_{\text {max }} \\
(8)\end{array}$ \\
\hline 1 & 2XMMi J001716.8-010725 & 1.163 & 2 & -12.72 & 45.06 & 1.10 & 1.10 \\
\hline 2 & 2XMMi J001808.7-005709 & 1.335 & 2 & -13.39 & 44.53 & 1.06 & 1.06 \\
\hline 3 & 2XMM J020011.5-093125 & 0.3604 & 2 & -12.49 & 44.11 & 2.15 & 2.15 \\
\hline 4 & 2XMM J020118.6-091936 & 0.6607 & 2 & -12.73 & 44.48 & 2.06 & 2.06 \\
\hline 5 & 2XMM J024040.8-081309 & 1.85 & 2 & -13.92 & 44.32 & 2.75 & 2.75 \\
\hline 6 & 2XMM J024055.8-081952 & 1.801 & 2 & -13.84 & 44.37 & 2.76 & 2.76 \\
\hline 7 & 2XMM J024105.8-081153 & 0.9785 & 2 & -13.66 & 43.95 & 2.91 & 2.91 \\
\hline 8 & 2XMM J024125.9-080936 & 3.072 & 2 & -14.13 & 44.60 & 2.60 & 2.60 \\
\hline 9 & 2XMM J024149.9-000433 & 1.26 & 2 & -13.46 & 44.40 & -0.43 & -0.43 \\
\hline 10 & 2XMM J024157.1+000703 & 1.563 & 2 & -13.46 & 44.62 & -0.48 & -0.48 \\
\hline 11 & 2XMM J024200.8+000021 & 1.104 & 2 & -12.85 & 44.88 & -0.39 & -0.39 \\
\hline 12 & 2XMM J024204.7+000814 & 0.3822 & 2 & -13.25 & 43.41 & -0.21 & -0.21 \\
\hline 13 & 2XMM J024207.2+000038 & 0.3842 & 2 & -13.06 & 43.61 & -0.21 & -0.21 \\
\hline 14 & 2XMM J024215.0-000209 & 1.01 & 2 & -13.62 & 44.02 & -0.37 & -0.37 \\
\hline 15 & 2XMM J024227.3+000846 & 0.6501 & 2 & -13.45 & 43.74 & -0.29 & -0.29 \\
\hline 16 & 2XMM J024250.8-000030 & 2.177 & 2 & -14.08 & 44.32 & -0.57 & -0.57 \\
\hline 17 & 2XMM J024251.0+001010 & 1.888 & 2 & -13.27 & 45.00 & -0.53 & -0.53 \\
\hline 18 & 2XMM J024304.6+000005 & 1.995 & 2 & -13.51 & 44.80 & -0.55 & -0.55 \\
\hline 19 & 2XMM J024308.1-000126 & 0.6787 & 2 & -13.67 & 43.57 & -0.30 & -0.30 \\
\hline 20 & 2XMM J030707.3-000424 & 0.6641 & 2 & -13.20 & 44.02 & 2.50 & 2.50 \\
\hline 21 & 2XMM J032108.4+413221 & 2.467 & 2 & -13.39 & 45.13 & 2.32 & 2.32 \\
\hline 22 & 2XMM J033627.4+004653 & 1.746 & 3 & -13.96 & 44.22 & 2.15 & 2.53 \\
\hline 23 & 2XMM J033639.5+002535 & 1.68 & 9 & -13.31 & 44.84 & -0.22 & 2.79 \\
\hline 24 & 2XMM J033654.2+004015 & 2.625 & 6 & -13.42 & 45.16 & -0.35 & 2.66 \\
\hline 25 & 2XMM J033701.1+004312 & 2.006 & 3 & -14.01 & 44.31 & 2.11 & 2.49 \\
\hline 26 & 2XMM J033709.1+004614 & 2.506 & 4 & -14.17 & 44.37 & -0.34 & 2.42 \\
\hline 27 & 2XMM J033711.5+004344 & 1.918 & 3 & -14.18 & 44.10 & 1.79 & 2.40 \\
\hline 28 & 2XMM J033715.6+004206 & 2.354 & 6 & -14.00 & 44.48 & -0.32 & 2.44 \\
\hline 29 & 2XMM J033716.5+003124 & 2.437 & 3 & -14.54 & 43.97 & 1.71 & 2.03 \\
\hline 30 & 2XMM J033718.8+003303 & 0.4371 & 7 & -13.80 & 42.99 & 0.05 & 2.81 \\
\hline 31 & 2XMM J033746.7+003510 & 1.4 & 2 & -13.78 & 44.19 & 0.81 & 0.81 \\
\hline 32 & 2XMM J033754.1+002934 & 2.004 & 2 & -13.43 & 44.89 & 2.39 & 2.39 \\
\hline 33 & 2XMM J033801.9+002719 & 1.583 & 2 & -13.68 & 44.41 & 2.45 & 2.45 \\
\hline 34 & 2XMM J073405.2+320315 & 2.082 & 2 & -14.13 & 44.23 & 1.71 & 1.71 \\
\hline 35 & 2XMMi J073654.0+302657 & 0.7238 & 2 & -13.13 & 44.17 & 1.99 & 1.99 \\
\hline 36 & 2XMMi J073708.1+303914 & 1.403 & 2 & -13.36 & 44.61 & 1.85 & 1.85 \\
\hline 37 & 2XMMi J073712.4+303637 & 0.9177 & 2 & -12.73 & 44.81 & 1.95 & 1.95 \\
\hline 38 & 2XMM J074222.3+494147 & 0.9274 & 6 & -12.97 & 44.58 & 1.22 & 3.16 \\
\hline 39 & 2XMM J080633.2+153810 & 0.9994 & 2 & -13.34 & 44.29 & 2.57 & 2.57 \\
\hline 40 & 2XMM J083102.9+523534 & 4.444 & 3 & -14.26 & 44.81 & 0.47 & 2.57 \\
\hline 41 & 2XMM J083740.2+245423 & 1.125 & 2 & -12.38 & 45.37 & 1.93 & 1.93 \\
\hline 42 & 2XMMi J083906.7+575417 & 1.534 & 2 & -12.23 & 45.83 & 1.94 & 1.94 \\
\hline 43 & 2XMMi J083924.8+575231 & 0.187 & 2 & -12.52 & 43.45 & 2.27 & 2.27 \\
\hline 44 & 2XMM J084659.3+344825 & 1.582 & 2 & -13.23 & 44.86 & 2.12 & 2.12 \\
\hline 45 & 2XMM J084710.0+345442 & 2.303 & 2 & -13.63 & 44.82 & 2.01 & 2.01 \\
\hline 46 & 2XMM J084905.0+445714 & 1.259 & 3 & -13.44 & 44.41 & -0.56 & 0.46 \\
\hline 47 & 2XMM J084943.6+450023 & 1.593 & 3 & -13.53 & 44.56 & -0.62 & 0.40 \\
\hline 48 & 2XMM J085346.1+200957 & 1.093 & 2 & -13.18 & 44.53 & 2.26 & 2.26 \\
\hline 49 & 2XMMi J090429.5+340544 & 1.297 & 2 & -13.67 & 44.22 & 2.19 & 2.19 \\
\hline 50 & 2XMMi J090505.5+341352 & 1.024 & 2 & -12.78 & 44.87 & 2.25 & 2.25 \\
\hline 51 & 2XMMi J090516.6+340921 & 1.872 & 2 & -13.35 & 44.90 & 2.09 & 2.09 \\
\hline 52 & 2XMMi J090525.2+341500 & 1.588 & 2 & -13.58 & 44.51 & 2.14 & 2.14 \\
\hline 53 & 2XMM J091301.0+525929 & 1.377 & 2 & -12.09 & 45.86 & 1.11 & 1.11 \\
\hline 54 & 2XMM J091302.8+530322 & 0.6307 & 2 & -13.98 & 43.18 & 1.28 & 1.28 \\
\hline 55 & 2XMM J091440.3+530038 & 1.43 & 2 & -13.60 & 44.38 & 1.11 & 1.11 \\
\hline 56 & 2XMM J091843.6+211819 & 0.8309 & 2 & -13.45 & 43.99 & 2.71 & 2.71 \\
\hline 57 & 2XMM J091848.6+211717 & 0.1493 & 2 & -12.47 & 43.29 & 2.91 & 2.91 \\
\hline 58 & 2XMM J091852.9+211518 & 1.035 & 2 & -14.33 & 43.33 & 2.66 & 2.66 \\
\hline 59 & 2XMM J091907.5+212553 & 1.39 & 2 & -12.90 & 45.06 & 2.59 & 2.59 \\
\hline 60 & 2XMM J091908.7+212153 & 1.514 & 2 & -13.32 & 44.72 & 2.57 & 2.57 \\
\hline 61 & 2XMM J091914.2+303018 & 1.388 & 2 & -14.11 & 43.84 & 2.78 & 2.78 \\
\hline 62 & 2XMM J092039.7+301701 & 1.18 & 2 & -14.00 & 43.79 & -0.98 & -0.98 \\
\hline 63 & 2XMM J092104.3+302031 & 3.35 & 3 & -13.80 & 45.01 & -1.28 & 2.52 \\
\hline 64 & 2XMM J093359.2+551550 & 1.863 & 2 & -13.26 & 44.99 & 0.33 & 0.33 \\
\hline 65 & 2XMM J093551.5+551117 & 1.79 & 2 & -13.63 & 44.58 & 0.34 & 0.34 \\
\hline
\end{tabular}


F. Vagnetti et al.: X-ray variability of AGNs

Table 1. continued.

\begin{tabular}{|c|c|c|c|c|c|c|c|}
\hline $\begin{array}{l}N_{\text {sou }} \\
\text { (1) }\end{array}$ & $\begin{array}{c}\text { Name } \\
\text { (2) }\end{array}$ & $\begin{array}{c}z \\
(3)\end{array}$ & $\begin{array}{l}N_{\text {epo }} \\
\text { (4) }\end{array}$ & $\log _{(5)} f_{X}$ & $\begin{array}{c}\log L_{X} \\
\text { (6) }\end{array}$ & $\begin{array}{c}\log \tau_{\min } \\
\text { (7) }\end{array}$ & $\begin{array}{c}\log \tau_{\text {max }} \\
\text { (8) }\end{array}$ \\
\hline 66 & 2XMM J093555.4+551238 & 1.8 & 2 & -13.33 & 44.89 & 0.34 & 0.34 \\
\hline 67 & 2XMM J094404.3+480647 & 0.3919 & 2 & -12.74 & 43.94 & 1.32 & 1.32 \\
\hline 68 & 2XMM J095251.5+013848 & 0.4997 & 2 & -13.39 & 43.53 & 2.05 & 2.05 \\
\hline 69 & 2XMM J095344.9+014251 & 1.657 & 2 & -13.22 & 44.91 & 1.80 & 1.80 \\
\hline 70 & 2XMM J095636.3+690028 & 1.975 & 3 & -13.47 & 44.84 & 0.30 & 2.48 \\
\hline 71 & 2XMM J095658.6+693852 & 2.035 & 2 & -13.38 & 44.96 & 2.55 & 2.55 \\
\hline 72 & 2XMM J095701.3+685500 & 1.297 & 3 & -13.07 & 44.82 & 0.42 & 2.59 \\
\hline 73 & 2XMMi J095750.0+013352 & 2.011 & 2 & -13.88 & 44.45 & 1.00 & 1.00 \\
\hline 74 & 2XMM J095754.7+023831 & 1.6 & 2 & -14.14 & 43.96 & 1.14 & 1.14 \\
\hline 75 & 2XMMi J095759.4+020435 & 2.034 & 3 & -13.81 & 44.53 & 0.96 & 2.09 \\
\hline 76 & 2XMM J095810.9+014004 & 2.101 & 3 & -13.64 & 44.73 & 0.99 & 2.10 \\
\hline 77 & 2XMM J095815.5+014922 & 1.509 & 6 & -12.92 & 45.12 & -1.01 & 2.20 \\
\hline 78 & 2XMM J095819.8+022903 & 0.3454 & 3 & -13.08 & 43.48 & 1.43 & 2.62 \\
\hline 79 & 2XMMi J095820.5+020303 & 1.355 & 3 & -14.26 & 43.67 & 1.11 & 2.20 \\
\hline 80 & 2XMM J095822.2+014524 & 1.96 & 5 & -13.57 & 44.73 & -1.08 & 2.13 \\
\hline 81 & 2XMM J095834.0+024427 & 1.887 & 2 & -13.46 & 44.80 & 2.09 & 2.09 \\
\hline 82 & 2XMMi J095834.7+014502 & 1.889 & 5 & -13.99 & 44.27 & -1.07 & 2.10 \\
\hline 83 & 2XMM J095844.9+014309 & 1.337 & 5 & -13.93 & 43.99 & 0.38 & 2.49 \\
\hline 84 & 2XMM J095847.7+690533 & 1.288 & 3 & -12.82 & 45.06 & 0.42 & 2.59 \\
\hline 85 & 2XMM J095848.8+023441 & 1.549 & 6 & -13.54 & 44.53 & -0.18 & 2.34 \\
\hline 86 & 2XMM J095852.1+025156 & 1.407 & 2 & -13.31 & 44.66 & 2.17 & 2.17 \\
\hline 87 & 2XMM J095857.3+021314 & 1.024 & 5 & -12.75 & 44.90 & 0.44 & 2.79 \\
\hline 88 & 2XMM J095858.6+020139 & 2.456 & 10 & -13.41 & 45.11 & -0.95 & 2.56 \\
\hline 89 & 2XMM J095902.7+021906 & 0.3454 & 9 & -12.61 & 43.95 & -0.72 & 2.97 \\
\hline 90 & 2XMM J095908.3+024309 & 1.318 & 2 & -12.72 & 45.18 & 2.18 & 2.18 \\
\hline 91 & 2XMM J095918.7+020951 & 1.156 & 7 & -12.85 & 44.93 & -0.09 & 2.76 \\
\hline 92 & 2ХMM J095924.4+015954 & 1.235 & 8 & -13.09 & 44.75 & -0.10 & 2.61 \\
\hline 93 & 2XMM J095935.6+024838 & 1.973 & 2 & -14.23 & 44.08 & 2.08 & 2.08 \\
\hline 94 & 2XMM J095946.0+024743 & 1.066 & 3 & -13.12 & 44.57 & 1.90 & 2.41 \\
\hline 95 & 2XMM J095949.4+020141 & 1.753 & 8 & -13.62 & 44.57 & -0.84 & 2.59 \\
\hline 96 & 2XMM J095958.0+014327 & 1.627 & 5 & -13.93 & 44.19 & -0.02 & 2.53 \\
\hline 97 & 2XMM J100001.3+024845 & 0.7661 & 3 & -13.20 & 44.16 & 1.97 & 2.47 \\
\hline 98 & 2XMM J100008.0+013307 & 1.172 & 4 & -13.80 & 43.99 & 1.91 & 2.62 \\
\hline 99 & 2XMM J100012.9+023522 & 0.6984 & 6 & -13.11 & 44.16 & 0.51 & 2.72 \\
\hline 100 & 2XMM J100014.1+020054 & 2.498 & 7 & -13.75 & 44.78 & -0.95 & 2.49 \\
\hline 101 & 2XMM J100024.3+015053 & 1.664 & 5 & -13.85 & 44.29 & 0.35 & 2.61 \\
\hline 102 & 2XMM J100024.6+023148 & 1.321 & 5 & -13.40 & 44.51 & 0.38 & 2.58 \\
\hline 103 & 2XMM J100025.2+015852 & 0.3726 & 7 & -12.53 & 44.11 & -0.54 & 2.90 \\
\hline 104 & 2XMM J100043.1+020637 & 0.36 & 10 & -13.23 & 43.37 & -0.54 & 2.90 \\
\hline 105 & 2XMM J100055.4+023442 & 1.404 & 8 & -13.53 & 44.43 & -0.97 & 2.58 \\
\hline 106 & 2XMM J100058.8+015359 & 1.557 & 10 & -13.55 & 44.53 & -1.11 & 2.63 \\
\hline 107 & 2XMM J100104.2+553522 & 1.535 & 2 & -13.31 & 44.74 & 2.56 & 2.56 \\
\hline 108 & 2XMM J100114.3+022356 & 1.796 & 6 & -13.56 & 44.65 & -1.04 & 2.41 \\
\hline 109 & 2XMM J100116.7+014053 & 2.054 & 4 & -13.75 & 44.59 & -0.12 & 2.25 \\
\hline 110 & 2XMM J100120.2+023341 & 1.834 & 5 & -13.81 & 44.43 & -1.04 & 2.51 \\
\hline 111 & 2XMM J100120.7+555351 & 1.413 & 2 & -11.98 & 45.99 & 2.58 & 2.58 \\
\hline 112 & 2XMM J100130.3+014304 & 1.57 & 4 & -14.04 & 44.04 & -0.83 & 2.15 \\
\hline 113 & 2XMM J100132.2+013419 & 1.355 & 3 & -13.78 & 44.15 & -0.79 & 2.19 \\
\hline 114 & 2XMM J100136.4+025304 & 2.116 & 3 & -13.54 & 44.84 & 1.79 & 2.37 \\
\hline 115 & 2XMM J100145.2+022456 & 2.032 & 3 & -14.00 & 44.33 & 0.90 & 2.09 \\
\hline 116 & 2XMM J100156.3+555440 & 1.152 & 2 & -13.33 & 44.44 & 2.63 & 2.63 \\
\hline 117 & 2XMM J100201.5+020330 & 2.023 & 6 & -14.20 & 44.12 & -0.42 & 2.27 \\
\hline 118 & 2XMM J100202.7+022434 & 0.9877 & 8 & -13.57 & 44.05 & 0.56 & 2.66 \\
\hline 119 & 2XMM J100205.2+554258 & 1.151 & 2 & -13.59 & 44.18 & 2.63 & 2.63 \\
\hline 120 & 2XMM J100210.6+023026 & 1.161 & 6 & -13.41 & 44.36 & -0.74 & 2.63 \\
\hline 121 & 2XMM J100219.5+015537 & 1.51 & 7 & -13.60 & 44.44 & -0.82 & 2.34 \\
\hline 122 & 2XMM J100226.3+021923 & 1.292 & 5 & -13.38 & 44.50 & 0.50 & 2.38 \\
\hline 123 & 2XMM J100232.1+023537 & 0.6576 & 4 & -13.06 & 44.14 & -0.62 & 2.52 \\
\hline 124 & 2XMM J100234.3+015011 & 1.504 & 4 & -13.37 & 44.67 & -0.81 & 2.16 \\
\hline 125 & 2XMM J100236.6+015949 & 1.516 & 2 & -14.07 & 43.97 & 1.84 & 1.84 \\
\hline 126 & 2XMM J100238.2+013747 & 2.506 & 2 & -13.63 & 44.91 & 2.02 & 2.02 \\
\hline 127 & 2XMM J100243.5+324812 & 0.7116 & 2 & -13.17 & 44.12 & 2.52 & 2.52 \\
\hline 128 & 2XMM J100248.9+325130 & 1.537 & 2 & -13.13 & 44.93 & 2.35 & 2.35 \\
\hline 129 & 2XMMi J100251.6+022905 & 2.006 & 2 & -13.81 & 44.51 & 2.26 & 2.26 \\
\hline 130 & 2XMM J100254.4+324039 & 0.8288 & 2 & -12.05 & 45.39 & 2.49 & 2.49 \\
\hline 131 & 2XMM J100302.9+015208 & 1.8 & 4 & -13.36 & 44.85 & -0.86 & 2.12 \\
\hline
\end{tabular}


Table 1. continued.

\begin{tabular}{|c|c|c|c|c|c|c|c|}
\hline $\begin{array}{l}N_{\text {sou }} \\
\text { (1) }\end{array}$ & $\begin{array}{l}\text { Name } \\
\text { (2) }\end{array}$ & $\begin{array}{c}z \\
(3)\end{array}$ & $\begin{array}{l}N_{\text {epo }} \\
\text { (4) }\end{array}$ & $\log _{(5)} f_{X}$ & $\begin{array}{c}\log L_{X} \\
(6)\end{array}$ & $\begin{array}{c}\log \tau_{\min } \\
\text { (7) }\end{array}$ & $\begin{array}{c}\log \tau_{\max } \\
\text { (8) }\end{array}$ \\
\hline 132 & 2XMM J100309.2+022037 & 1.964 & 3 & -13.91 & 44.39 & 0.39 & 2.12 \\
\hline 133 & 2XMM J100309.4+554134 & 0.6736 & 2 & -12.95 & 44.28 & 2.74 & 2.74 \\
\hline 134 & 2XMM J100324.5+021830 & 0.5184 & 2 & -12.79 & 44.17 & 2.40 & 2.40 \\
\hline 135 & 2XMM J100926.6+533424 & 1.73 & 2 & -13.02 & 45.16 & 2.94 & 2.94 \\
\hline 136 & 2XMMi J102129.1+215609 & 1.465 & 2 & -13.35 & 44.67 & 1.82 & 1.82 \\
\hline 137 & 2XMMi J102134.2+215437 & 1.536 & 2 & -13.49 & 44.57 & 1.81 & 1.81 \\
\hline 138 & 2XMMi J102223.7+383424 & 1.357 & 2 & -13.62 & 44.31 & 0.82 & 0.82 \\
\hline 139 & 2XMMi J102224.0+215832 & 1.165 & 2 & -13.64 & 44.14 & 1.88 & 1.88 \\
\hline 140 & 2XMMi J102255.3+383007 & 0.658 & 2 & -13.57 & 43.64 & 0.98 & 0.98 \\
\hline 141 & 2XMM J102310.0+194248 & 1.594 & 2 & -13.64 & 44.45 & 2.93 & 2.93 \\
\hline 142 & 2XMM J102313.2+195651 & 1.086 & 2 & -13.13 & 44.58 & 3.02 & 3.02 \\
\hline 143 & 2XMM J102318.6+194835 & 1.761 & 2 & -13.77 & 44.42 & 2.90 & 2.90 \\
\hline 144 & 2XMM J102423.7+195250 & 1.635 & 2 & -13.83 & 44.29 & 2.92 & 2.92 \\
\hline 145 & 2XMM J103216.0+505119 & 0.1731 & 4 & -12.44 & 43.46 & 0.71 & 3.03 \\
\hline 146 & 2XMM J103227.9+573822 & 1.969 & 2 & -13.23 & 45.07 & 1.15 & 1.15 \\
\hline 147 & 2XMM J103518.5+392934 & 0.8774 & 2 & -13.61 & 43.89 & 3.00 & 3.00 \\
\hline 148 & 2XMM J104401.1+212804 & 1.494 & 3 & -13.17 & 44.86 & 0.98 & 1.95 \\
\hline 149 & 2XMM J104414.5+213203 & 1.17 & 3 & -13.65 & 44.13 & 1.04 & 2.01 \\
\hline 150 & 2XMM J104440.0+212643 & 1.504 & 3 & -13.34 & 44.70 & 0.97 & 1.95 \\
\hline 151 & 2XMM J104522.1+212614 & 0.8908 & 3 & -13.07 & 44.44 & 1.10 & 2.07 \\
\hline 152 & 2XMM J105039.5+572336 & 1.445 & 3 & -13.35 & 44.64 & -0.09 & 0.39 \\
\hline 153 & 2XMM J105050.0+573819 & 1.285 & 3 & -13.33 & 44.55 & -0.06 & 0.42 \\
\hline 154 & 2XMM J105201.3+441417 & 1.79 & 2 & -13.72 & 44.48 & 1.02 & 1.02 \\
\hline 155 & 2XMM J105204.5+440152 & 1.524 & 2 & -13.47 & 44.58 & 1.06 & 1.06 \\
\hline 156 & 2XMM J105221.0+440439 & 0.9677 & 2 & -13.16 & 44.43 & 1.17 & 1.17 \\
\hline 157 & 2XMM J105224.9+441505 & 0.4435 & 2 & -12.48 & 44.33 & 1.31 & 1.31 \\
\hline 158 & 2XMM J105239.6+572431 & 1.112 & 14 & -12.90 & 44.83 & -0.05 & 2.65 \\
\hline 159 & 2XMM J105316.7+573550 & 1.205 & 13 & -12.78 & 45.03 & -0.09 & 2.64 \\
\hline 160 & 2XMM J105404.1+574019 & 1.102 & 3 & -13.45 & 44.27 & -0.07 & 0.26 \\
\hline 161 & 2XMM J105422.5+572031 & 2.972 & 3 & -13.94 & 44.76 & -0.35 & -0.02 \\
\hline 162 & 2XMMi J105540.0+065552 & 0.596 & 5 & -13.46 & 43.64 & 0.08 & 2.01 \\
\hline 163 & 2XMMi J105549.4+065542 & 0.9024 & 5 & -12.77 & 44.75 & 0.01 & 1.93 \\
\hline 164 & 2XMMi J105603.7+070235 & 2.303 & 5 & -13.38 & 45.07 & -0.23 & 1.70 \\
\hline 165 & 2XMMi J105622.1+071250 & 1.656 & 5 & -13.09 & 45.05 & -0.14 & 1.79 \\
\hline 166 & 2XMM J110253.4+360425 & 1.795 & 2 & -13.90 & 44.31 & 2.59 & 2.59 \\
\hline 167 & 2XMM J110309.2+380914 & 1.721 & 2 & -13.44 & 44.73 & 1.02 & 1.02 \\
\hline 168 & 2XMM J110320.1+380931 & 1.752 & 12 & -13.76 & 44.43 & -0.87 & 2.83 \\
\hline 169 & 2XMM J110334.7+355108 & 1.199 & 2 & -13.75 & 44.06 & 2.70 & 2.70 \\
\hline 170 & 2XMM J110400.3+380231 & 1.621 & 21 & -13.74 & 44.37 & -1.31 & 3.05 \\
\hline 171 & 2XMM J110449.0+381812 & 1.943 & 25 & -13.54 & 44.75 & -1.36 & 3.05 \\
\hline 172 & 2XMM J110458.2+250422 & 3.574 & 2 & -13.48 & 45.39 & 2.20 & 2.20 \\
\hline 173 & 2XMM J110547.1+380948 & 1.153 & 6 & -13.12 & 44.65 & -1.22 & 2.93 \\
\hline 174 & 2XMM J110550.6+251747 & 0.6247 & 2 & -12.53 & 44.62 & 2.65 & 2.65 \\
\hline 175 & 2XMM J110602.6+251227 & 1.683 & 2 & -13.48 & 44.66 & 2.43 & 2.43 \\
\hline 176 & 2XMMi J111233.4+060619 & 3.278 & 2 & -14.09 & 44.70 & -0.36 & -0.36 \\
\hline 177 & 2XMMi J111303.6+061620 & 0.8491 & 2 & -13.16 & 44.30 & 0.00 & 0.00 \\
\hline 178 & 2XMM J111506.0+424949 & 0.3005 & 2 & -13.16 & 43.27 & 2.83 & 2.83 \\
\hline 179 & 2XMM J111747.3+075400 & 1.961 & 3 & -13.88 & 44.42 & 0.72 & 2.50 \\
\hline 180 & 2XMM J111816.9+074558 & 1.735 & 3 & -12.42 & 45.76 & 0.76 & 2.54 \\
\hline 181 & 2XMM J111840.5+075323 & 1.461 & 3 & -13.37 & 44.63 & 0.80 & 2.58 \\
\hline 182 & 2XMM J111842.3+212014 & 1.924 & 2 & -13.61 & 44.67 & 2.58 & 2.58 \\
\hline 183 & 2XMM J111853.4+074946 & 2.042 & 3 & -13.66 & 44.68 & 0.71 & 2.49 \\
\hline 184 & 2XMM J111902.0+213315 & 1.933 & 2 & -13.39 & 44.90 & 2.58 & 2.58 \\
\hline 185 & 2XMM J111928.3+130250 & 2.394 & 2 & -12.81 & 45.68 & 2.03 & 2.03 \\
\hline 186 & 2XMM J113205.1+530726 & 1.84 & 2 & -13.74 & 44.50 & 1.82 & 1.82 \\
\hline 187 & 2XMM J113224.0+525157 & 0.837 & 2 & -13.38 & 44.07 & 2.00 & 2.00 \\
\hline 188 & 2XMM J114405.6+195734 & 0.9541 & 2 & -13.06 & 44.52 & 1.96 & 1.96 \\
\hline 189 & 2XMM J115606.7+233106 & 1.593 & 3 & -12.90 & 45.19 & 0.79 & 2.99 \\
\hline 190 & 2XMM J115726.2+434954 & 1.597 & 2 & -13.23 & 44.87 & -0.16 & -0.16 \\
\hline 191 & 2XMM J115838.5+435505 & 1.208 & 2 & -13.63 & 44.18 & -0.09 & -0.09 \\
\hline 192 & 2XMM J115851.0+435048 & 0.2871 & 2 & -12.92 & 43.46 & 0.14 & 0.14 \\
\hline 193 & 2XMM J115906.3+434643 & 1.462 & 2 & -13.50 & 44.51 & -0.14 & -0.14 \\
\hline 194 & 2XMM J115911.3+440819 & 1.438 & 2 & -13.50 & 44.50 & -0.13 & -0.13 \\
\hline 195 & 2XMM J120405.8+201345 & 0.5985 & 3 & -12.83 & 44.27 & 1.10 & 2.05 \\
\hline 196 & 2XMM J120414.4+351759 & 2.359 & 2 & -13.86 & 44.62 & 1.05 & 1.05 \\
\hline 197 & 2XMM J120432.7+202434 & 2.09 & 3 & -13.34 & 45.02 & 0.81 & 1.76 \\
\hline
\end{tabular}


F. Vagnetti et al.: X-ray variability of AGNs

Table 1. continued.

\begin{tabular}{|c|c|c|c|c|c|c|c|}
\hline $\begin{array}{l}N_{\text {sou }} \\
\text { (1) }\end{array}$ & $\begin{array}{l}\text { Name } \\
\text { (2) }\end{array}$ & $\begin{array}{c}z \\
(3)\end{array}$ & $\begin{array}{c}N_{\text {epo }} \\
\text { (4) }\end{array}$ & $\begin{array}{c}\log f_{X} \\
(5)\end{array}$ & $\begin{array}{c}\log L_{X} \\
\text { (6) }\end{array}$ & $\begin{array}{c}\log \tau_{\min } \\
\text { (7) }\end{array}$ & $\begin{array}{c}\log \tau_{\max } \\
\text { (8) }\end{array}$ \\
\hline 198 & 2XMM J120504.4+352209 & 2.278 & 2 & -13.64 & 44.80 & 1.06 & 1.06 \\
\hline 199 & 2XMM J120943.4+393644 & 2.333 & 2 & -13.53 & 44.94 & -0.79 & -0.79 \\
\hline 200 & 2XMM J121001.7+392151 & 2.955 & 3 & -13.66 & 45.03 & -0.86 & 2.70 \\
\hline 201 & 2XMM J121111.1+393320 & 1.529 & 8 & -13.68 & 44.37 & -0.81 & 2.93 \\
\hline 202 & 2XMM J121129.3+392513 & 1.671 & 8 & -13.70 & 44.45 & -0.84 & 2.91 \\
\hline 203 & 2XMM J121426.5+140259 & 1.279 & 4 & -12.83 & 45.05 & -0.04 & 3.02 \\
\hline 204 & 2XMM J121509.4+135450 & 0.8473 & 2 & -12.76 & 44.70 & 2.78 & 2.78 \\
\hline 205 & 2XMM J121753.1+294305 & 1.647 & 8 & -13.28 & 44.85 & -0.13 & 2.84 \\
\hline 206 & 2XMM J121808.5+471613 & 0.398 & 7 & -12.75 & 43.95 & 1.48 & 3.19 \\
\hline 207 & 2XMM J121836.1+054628 & 0.7954 & 2 & -13.08 & 44.32 & 3.09 & 3.09 \\
\hline 208 & 2XMM J121849.5+295451 & 0.962 & 8 & -13.21 & 44.38 & 0.00 & 2.97 \\
\hline 209 & 2XMM J121911.1+470708 & 1.901 & 5 & -13.60 & 44.66 & 1.71 & 2.87 \\
\hline 210 & 2XMM J121938.6+064022 & 1.187 & 2 & -13.23 & 44.57 & 1.88 & 1.88 \\
\hline 211 & 2XMM J121952.2+472058 & 0.6531 & 6 & -12.86 & 44.34 & 1.40 & 3.12 \\
\hline 212 & 2XMMi J122051.4+282217 & 1.524 & 4 & -13.58 & 44.47 & -0.20 & 0.37 \\
\hline 213 & 2XMM J122135.6+280614 & 3.288 & 5 & -12.70 & 46.09 & -0.43 & 2.71 \\
\hline 214 & 2XMM J122222.7+041623 & 1.19 & 2 & -13.54 & 44.27 & 2.53 & 2.53 \\
\hline 215 & 2XMM J122442.2+332941 & 0.7763 & 3 & -13.02 & 44.35 & 2.09 & 2.49 \\
\hline 216 & 2XMM J122525.0+333651 & 0.7654 & 3 & -13.21 & 44.14 & 2.09 & 2.49 \\
\hline 217 & 2XMM J122532.4+332532 & 0.5859 & 3 & -12.87 & 44.22 & 2.14 & 2.54 \\
\hline 218 & 2XMM J122549.9+332455 & 1.133 & 2 & -13.82 & 43.93 & 2.41 & 2.41 \\
\hline 219 & 2XMM J122556.1+130656 & 1.35 & 2 & -13.48 & 44.45 & 2.59 & 2.59 \\
\hline 220 & 2XMM J122607.1+334559 & 1.158 & 3 & -12.96 & 44.82 & 2.00 & 2.41 \\
\hline 221 & 2XMM J122627.0+332148 & 0.875 & 3 & -13.67 & 43.83 & 2.07 & 2.47 \\
\hline 222 & $2 X M M ~ J 122645.3+332801$ & 3.339 & 2 & -14.00 & 44.81 & 1.63 & 1.63 \\
\hline 223 & $2 \mathrm{XMM} \mathrm{J} 122703.3+125402$ & 1.273 & 2 & -13.60 & 44.27 & 2.60 & 2.60 \\
\hline 224 & 2XMM J122731.6+333259 & 1.608 & 2 & -13.93 & 44.17 & 2.62 & 2.62 \\
\hline 225 & 2XMM J122923.7+075359 & 0.8538 & 2 & -12.95 & 44.52 & 2.49 & 2.49 \\
\hline 226 & 2XMM J122931.2+015249 & 0.7704 & 19 & -13.57 & 43.79 & -0.81 & 3.24 \\
\hline 227 & 2XMM J122934.7+015658 & 1.921 & 25 & -13.29 & 44.99 & -1.43 & 3.03 \\
\hline 228 & 2XMM J122951.5+105827 & 1.847 & 2 & -13.65 & 44.59 & 0.14 & 0.14 \\
\hline 229 & 2XMM J123035.4+153510 & 0.8028 & 2 & -12.84 & 44.57 & 0.30 & 0.30 \\
\hline 230 & 2XMM J123049.7+640848 & 1.041 & 2 & -13.57 & 44.10 & 2.72 & 2.72 \\
\hline 231 & 2XMM J123054.1+110011 & 0.2359 & 3 & -11.74 & 44.45 & 0.50 & 2.86 \\
\hline 232 & 2XMM J123110.3+161258 & 1.453 & 2 & -13.05 & 44.95 & -1.02 & -1.02 \\
\hline 233 & 2XMM J123126.4+105111 & 0.3039 & 3 & -12.76 & 43.68 & 0.48 & 2.83 \\
\hline 234 & 2XMM J123147.1+123835 & 0.2916 & 2 & -12.69 & 43.71 & 3.29 & 3.29 \\
\hline 235 & 2XMM J123148.0+143741 & 1.706 & 2 & -13.37 & 44.80 & 1.90 & 1.90 \\
\hline 236 & 2XMM J123229.6+641115 & 0.7423 & 2 & -12.98 & 44.34 & 2.79 & 2.79 \\
\hline 237 & 2XMM J123622.9+621526 & 2.588 & 7 & -13.96 & 44.61 & -0.80 & 2.42 \\
\hline 238 & 2XMM J123716.0+620323 & 2.068 & 5 & -14.31 & 44.04 & 0.24 & 2.49 \\
\hline 239 & 2XMM J123759.5+621102 & 0.9095 & 7 & -13.05 & 44.48 & -0.52 & 2.69 \\
\hline 240 & 2XMM J123800.9+621336 & 0.4402 & 7 & -13.21 & 43.60 & -0.40 & 2.81 \\
\hline 241 & 2XMM J123816.0+620208 & 1.005 & 3 & -14.01 & 43.62 & -0.54 & 0.85 \\
\hline 242 & 2XMM J124126.5+323924 & 1.787 & 2 & -13.50 & 44.70 & 0.95 & 0.95 \\
\hline 243 & 2XMM J124206.0+141920 & 1.951 & 2 & -13.26 & 45.03 & 2.57 & 2.57 \\
\hline 244 & 2XMM J124207.6+333117 & 0.5148 & 2 & -13.38 & 43.58 & 0.07 & 0.07 \\
\hline 245 & 2XMM J124300.3+113554 & 2.94 & 2 & -13.12 & 45.57 & 2.81 & 2.81 \\
\hline 246 & 2XMM J124406.9+113524 & 1.344 & 2 & -13.07 & 44.85 & 3.04 & 3.04 \\
\hline 247 & 2XMM J125317.6+310550 & 0.7824 & 2 & -13.40 & 43.98 & 1.22 & 1.22 \\
\hline 248 & 2XMM J125344.9+305820 & 2.067 & 2 & -13.42 & 44.93 & 0.98 & 0.98 \\
\hline 249 & 2XMM J125553.0+272405 & 0.3158 & 2 & -12.22 & 44.25 & 2.92 & 2.92 \\
\hline 250 & 2XMM J125627.9+215406 & 1.871 & 5 & -13.29 & 44.96 & 0.77 & 2.41 \\
\hline 251 & 2XMM J125629.6+271507 & 2.523 & 2 & -13.56 & 44.98 & 2.49 & 2.49 \\
\hline 252 & 2XMM J125702.9+273801 & 1.13 & 3 & -13.35 & 44.40 & 0.67 & 1.93 \\
\hline 253 & 2XMM J125708.4+271330 & 1.664 & 3 & -13.27 & 44.86 & 0.15 & 2.91 \\
\hline 254 & $2 \mathrm{XMM} \mathrm{J} 125712.0+274216$ & 0.7925 & 2 & -13.03 & 44.37 & 2.01 & 2.01 \\
\hline 255 & 2XMM J125732.6+215708 & 1.934 & 4 & -13.69 & 44.60 & 1.78 & 2.40 \\
\hline 256 & 2XMMi J125732.9+473224 & 1.859 & 2 & -13.57 & 44.67 & -0.18 & -0.18 \\
\hline 257 & 2XMM J125745.1+273210 & 1.56 & 5 & -13.77 & 44.30 & 0.17 & 2.93 \\
\hline 258 & $2 \mathrm{XMM} \mathrm{J} 125803.0+345125$ & 2.037 & 2 & -14.01 & 44.33 & 0.93 & 0.93 \\
\hline 259 & 2XMM J125818.5+275937 & 1.723 & 2 & -13.91 & 44.26 & 1.82 & 1.82 \\
\hline 260 & 2XMM J125831.7+275330 & 1.14 & 5 & -13.04 & 44.72 & -0.82 & 2.93 \\
\hline 261 & 2XMM J125859.2+275308 & 1.135 & 12 & -13.30 & 44.45 & -0.82 & 2.94 \\
\hline 262 & 2XMM J125903.9+344702 & 0.6075 & 3 & -13.01 & 44.12 & 1.20 & 2.03 \\
\hline 263 & 2XMM J125923.3+272720 & 1.99 & 2 & -13.46 & 44.85 & 0.15 & 0.15 \\
\hline
\end{tabular}


Table 1. continued.

\begin{tabular}{|c|c|c|c|c|c|c|c|}
\hline $\begin{array}{l}N_{\text {sou }} \\
\text { (1) }\end{array}$ & $\begin{array}{l}\text { Name } \\
\text { (2) }\end{array}$ & $\begin{array}{c}z \\
(3)\end{array}$ & $\begin{array}{l}N_{\text {epo }} \\
\text { (4) }\end{array}$ & $\log _{(5)} f_{X}$ & $\begin{array}{c}\log L_{X} \\
(6)\end{array}$ & $\begin{array}{c}\log \tau_{\min } \\
\text { (7) }\end{array}$ & $\begin{array}{c}\log \tau_{\text {max }} \\
\text { (8) }\end{array}$ \\
\hline 264 & 2XMM J125931.0+282706 & 1.094 & 7 & -13.70 & 44.02 & -0.06 & 3.02 \\
\hline 265 & 2XMM J130002.7+345043 & 1.054 & 3 & -13.25 & 44.43 & 1.10 & 1.92 \\
\hline 266 & 2XMM J130028.5+283010 & 0.6467 & 7 & -12.09 & 45.10 & 0.05 & 2.95 \\
\hline 267 & 2XMM J130048.1+282321 & 1.923 & 7 & -13.42 & 44.86 & -0.20 & 2.70 \\
\hline 268 & 2XMM J130100.8+281944 & 1.36 & 7 & -13.09 & 44.85 & -0.11 & 2.79 \\
\hline 269 & 2XMM J130120.0+282137 & 1.369 & 8 & -12.73 & 45.21 & -0.11 & 2.79 \\
\hline 270 & 2XMMi J131134.9+231818 & 1.527 & 2 & -13.50 & 44.55 & 0.52 & 0.52 \\
\hline 271 & 2XMMi J131213.6+231958 & 1.514 & 3 & -13.10 & 44.94 & -0.17 & 0.60 \\
\hline 272 & 2XMMi J131236.2+231630 & 3.711 & 2 & -13.86 & 45.04 & 0.24 & 0.24 \\
\hline 273 & 2XMMi J131606.6+421513 & 1.841 & 2 & -13.32 & 44.92 & 0.89 & 0.89 \\
\hline 274 & 2XMMi J131712.9+420439 & 1.031 & 2 & -12.78 & 44.88 & 1.03 & 1.03 \\
\hline 275 & 2XMM J132307.7+655446 & 0.6485 & 2 & -12.91 & 44.28 & 2.64 & 2.64 \\
\hline 276 & 2XMM J132827.3+581839 & 3.139 & 4 & -14.07 & 44.68 & 0.01 & 2.54 \\
\hline 277 & 2XMM J132938.5+471854 & 1.027 & 4 & -13.27 & 44.39 & 0.31 & 2.78 \\
\hline 278 & 2XMM J133028.3+242253 & 1.919 & 2 & -13.66 & 44.62 & 0.64 & 0.64 \\
\hline 279 & 2XMM J133114.5+241650 & 2.265 & 2 & -13.72 & 44.71 & 0.59 & 0.59 \\
\hline 280 & 2XMM J133342.3+380336 & 1.077 & 3 & -13.16 & 44.54 & -0.09 & 0.75 \\
\hline 281 & 2XMM J133417.5+375722 & 1.142 & 3 & -13.25 & 44.51 & -0.10 & 0.74 \\
\hline 282 & 2XMM J133542.5+375542 & 1.899 & 3 & -13.84 & 44.43 & -0.23 & 0.61 \\
\hline 283 & 2XMM J133807.5+242410 & 0.6313 & 4 & -12.97 & 44.20 & 0.07 & 3.16 \\
\hline 284 & 2XMM J133859.2+272702 & 1.792 & 2 & -13.62 & 44.58 & 1.17 & 1.17 \\
\hline 285 & 2XMM J133913.3+271818 & 0.6819 & 2 & -12.71 & 44.53 & 1.39 & 1.39 \\
\hline 286 & 2XMM J133944.4-001451 & 1.269 & 2 & -13.82 & 44.05 & 1.92 & 1.92 \\
\hline 287 & 2XMMi J134050.7+301610 & 1.519 & 2 & -13.52 & 44.53 & -0.10 & -0.10 \\
\hline 288 & 2XMMi J134132.8+301326 & 0.7355 & 2 & -13.20 & 44.12 & 0.06 & 0.06 \\
\hline 289 & 2XMM J134256.5+000057 & 0.8041 & 2 & -12.76 & 44.65 & 2.48 & 2.48 \\
\hline 290 & 2XMM J134323.6+001223 & 0.8731 & 3 & -12.79 & 44.70 & 1.93 & 2.47 \\
\hline 291 & 2XMM J134834.2+262205 & 0.9144 & 2 & -12.67 & 44.86 & 2.69 & 2.69 \\
\hline 292 & 2XMM J134848.2+262219 & 0.5949 & 2 & -12.93 & 44.17 & 2.77 & 2.77 \\
\hline 293 & 2XMM J134850.1+262503 & 2.915 & 2 & -14.13 & 44.55 & 2.38 & 2.38 \\
\hline 294 & 2XMM J135038.6+601901 & 1.165 & 2 & -13.49 & 44.29 & 1.98 & 1.98 \\
\hline 295 & 2XMM J135301.2+633256 & 3.16 & 2 & -14.26 & 44.49 & 2.54 & 2.54 \\
\hline 296 & 2XMM J135418.1+635705 & 1.618 & 3 & -13.78 & 44.33 & -0.11 & 2.74 \\
\hline 297 & 2XMM J135810.6+653740 & 1.112 & 3 & -13.07 & 44.66 & 1.25 & 1.58 \\
\hline 298 & 2XMM J135842.7+652236 & 3.199 & 3 & -13.81 & 44.95 & 0.95 & 1.28 \\
\hline 299 & 2XMM J140001.6-014924 & 1.754 & 3 & -13.40 & 44.79 & 1.83 & 2.67 \\
\hline 300 & 2XMM J140146.5+024433 & 4.442 & 4 & -13.69 & 45.39 & 0.26 & 2.73 \\
\hline 301 & 2XMMi J140148.2-014514 & 1.795 & 2 & -13.78 & 44.44 & 1.82 & 1.82 \\
\hline 302 & 2XMM J140349.4+432009 & 0.6664 & 2 & -12.94 & 44.28 & 1.49 & 1.49 \\
\hline 303 & 2XMM J140354.6+543246 & 3.258 & 2 & -14.07 & 44.71 & 2.26 & 2.26 \\
\hline 304 & 2XMM J140536.6+255140 & 0.9427 & 3 & -13.01 & 44.55 & 0.04 & 2.96 \\
\hline 305 & 2XMM J140541.0+432537 & 0.5199 & 2 & -13.23 & 43.74 & 1.53 & 1.53 \\
\hline 306 & 2XMMi J140547.2+260629 & 0.7244 & 2 & -13.60 & 43.71 & 0.09 & 0.09 \\
\hline 307 & 2XMM J140841.5+262943 & 1.885 & 2 & -13.48 & 44.78 & 2.06 & 2.06 \\
\hline 308 & 2XMM J140949.0+261347 & 2.945 & 2 & -13.94 & 44.75 & 1.93 & 1.93 \\
\hline 309 & 2XMM J141513.5+112216 & 1.554 & 2 & -13.71 & 44.36 & 2.16 & 2.16 \\
\hline 310 & 2XMM J141515.8+112344 & 1.229 & 2 & -13.48 & 44.36 & 2.22 & 2.22 \\
\hline 311 & 2XMM J141540.0+112407 & 1.074 & 2 & -13.02 & 44.68 & 2.25 & 2.25 \\
\hline 312 & 2XMM J141546.2+112943 & 2.56 & 2 & -13.74 & 44.82 & 2.02 & 2.02 \\
\hline 313 & 2XMM J141551.6+522743 & 2.587 & 2 & -14.08 & 44.49 & -0.30 & -0.30 \\
\hline 314 & 2XMM J141642.3+521813 & 1.285 & 3 & -13.68 & 44.20 & -0.98 & -0.11 \\
\hline 315 & 2XMM J141647.3+521115 & 2.152 & 3 & -13.56 & 44.83 & -1.12 & -0.25 \\
\hline 316 & 2XMM J141652.0+113201 & 0.6881 & 2 & -13.00 & 44.25 & 2.34 & 2.34 \\
\hline 317 & 2XMM J141745.6+250242 & 1.36 & 3 & -13.61 & 44.32 & -0.00 & 1.93 \\
\hline 318 & 2XMM J141838.2+522400 & 1.118 & 3 & -13.23 & 44.51 & -0.94 & -0.07 \\
\hline 319 & 2XMMi J142258.2+193322 & 1.603 & 3 & -12.65 & 45.45 & -0.12 & 0.19 \\
\hline 320 & 2XMMi J142259.6+194458 & 1.129 & 3 & -13.71 & 44.04 & -0.03 & 0.27 \\
\hline 321 & 2XMM J142325.4+384032 & 0.2489 & 2 & -12.47 & 43.78 & 2.73 & 2.73 \\
\hline 322 & 2XMM J142335.9+383407 & 1.487 & 2 & -13.60 & 44.42 & 2.43 & 2.43 \\
\hline 323 & 2XMM J142355.5+383150 & 1.207 & 2 & -13.08 & 44.74 & 2.49 & 2.49 \\
\hline 324 & 2XMM J142406.6+383714 & 1.562 & 2 & -13.11 & 44.96 & 2.42 & 2.42 \\
\hline 325 & 2XMM J142435.9+421030 & 2.218 & 2 & -13.11 & 45.30 & 1.65 & 1.65 \\
\hline 326 & 2XMM J142455.5+421408 & 0.3162 & 2 & -12.03 & 44.45 & 2.04 & 2.04 \\
\hline 327 & 2XMM J142519.0+422158 & 1.104 & 2 & -13.45 & 44.28 & 1.83 & 1.83 \\
\hline 328 & 2XMM J142737.7+424450 & 1.953 & 7 & -13.55 & 44.74 & -0.17 & 2.71 \\
\hline 329 & 2XMM J143025.8+415957 & 0.3524 & 3 & -12.87 & 43.71 & 1.46 & 2.83 \\
\hline
\end{tabular}


F. Vagnetti et al.: X-ray variability of AGNs

Table 1. continued.

\begin{tabular}{|c|c|c|c|c|c|c|c|}
\hline $\begin{array}{l}N_{\text {sou }} \\
\text { (1) }\end{array}$ & $\begin{array}{l}\text { Name } \\
\text { (2) }\end{array}$ & $\begin{array}{c}z \\
(3)\end{array}$ & $\begin{array}{l}N_{\text {epo }} \\
\text { (4) }\end{array}$ & $\log _{(5)} f_{\mathrm{X}}$ & $\begin{array}{c}\log L_{X} \\
\text { (6) }\end{array}$ & $\begin{array}{c}\log \tau_{\text {min }} \\
(7)\end{array}$ & $\begin{array}{c}\log \tau_{\text {max }} \\
\text { (8) }\end{array}$ \\
\hline 330 & 2XMM J143440.4+484139 & 1.945 & 2 & -13.50 & 44.79 & 1.01 & 1.01 \\
\hline 331 & 2XMM J143513.9+484149 & 1.887 & 2 & -13.48 & 44.78 & 1.02 & 1.02 \\
\hline 332 & 2XMM J143621.2+484606 & 2.395 & 2 & -13.61 & 44.88 & 0.95 & 0.95 \\
\hline 333 & 2XMMi J143914.1+002320 & 0.8826 & 2 & -13.05 & 44.45 & 1.99 & 1.99 \\
\hline 334 & 2XMMi J143931.9+000453 & 1.405 & 2 & -13.33 & 44.63 & 1.89 & 1.89 \\
\hline 335 & 2XMMi J144008.6+001630 & 1.502 & 2 & -13.08 & 44.95 & 1.87 & 1.87 \\
\hline 336 & 2XMMi J144259.9-003725 & 1.817 & 2 & -12.93 & 45.29 & -0.43 & -0.43 \\
\hline 337 & 2XMMi J144305.1-004825 & 0.7007 & 2 & -13.68 & 43.59 & -0.22 & -0.22 \\
\hline 338 & 2XMMi J144308.1-004913 & 1.372 & 2 & -12.97 & 44.97 & -0.36 & -0.36 \\
\hline 339 & 2XMM J144729.9+030520 & 1.782 & 2 & -13.47 & 44.73 & 2.10 & 2.10 \\
\hline 340 & 2XMM J150424.9+102938 & 1.839 & 4 & -12.31 & 45.92 & -1.17 & 2.76 \\
\hline 341 & 2XMM J150428.3+101856 & 1.011 & 4 & -12.95 & 44.69 & -1.02 & 2.91 \\
\hline 342 & 2XMM J150545.6+014145 & 1.424 & 2 & -13.22 & 44.76 & 1.94 & 1.94 \\
\hline 343 & 2XMM J150916.2+332730 & 1.656 & 2 & -13.46 & 44.67 & 2.52 & 2.52 \\
\hline 344 & 2XMM J150948.6+333626 & 0.5124 & 2 & -13.17 & 43.78 & 2.77 & 2.77 \\
\hline 345 & 2XMM J151126.4+565934 & 1.031 & 2 & -13.45 & 44.21 & 2.12 & 2.12 \\
\hline 346 & 2XMM J151453.9+561032 & 1.287 & 2 & -13.21 & 44.67 & 0.54 & 0.54 \\
\hline 347 & 2XMM J151510.1+562834 & 0.7207 & 2 & -13.22 & 44.07 & 0.67 & 0.67 \\
\hline 348 & 2XMM J151651.2+562850 & 1.309 & 2 & -13.35 & 44.55 & 0.54 & 0.54 \\
\hline 349 & 2XMM J152322.3+274931 & 0.424 & 2 & -13.90 & 42.87 & 2.80 & 2.80 \\
\hline 350 & 2XMM J152553.8+513649 & 2.883 & 3 & -12.83 & 45.84 & 0.03 & 1.54 \\
\hline 351 & 2XMM J153322.8+324351 & 1.899 & 3 & -13.53 & 44.74 & 1.72 & 2.48 \\
\hline 352 & 2XMMi J153434.8+574723 & 1.236 & 4 & -13.53 & 44.31 & -0.06 & 0.42 \\
\hline 353 & 2XMMi J153458.3+575625 & 1.129 & 4 & -13.23 & 44.52 & -0.03 & 0.45 \\
\hline 354 & 2XMM J153617.2+544709 & 1.45 & 4 & -13.15 & 44.85 & -0.08 & 2.68 \\
\hline 355 & 2XMM J153634.9+544317 & 0.9136 & 4 & -13.23 & 44.30 & 0.02 & 2.79 \\
\hline 356 & 2XMM J153641.5+543505 & 0.447 & 4 & -13.54 & 43.28 & 0.14 & 2.91 \\
\hline 357 & 2XMMi J153716.2+574838 & 0.6406 & 4 & -12.95 & 44.23 & 0.08 & 0.56 \\
\hline 358 & 2XMM J154234.3+540137 & 0.3959 & 2 & -13.77 & 42.93 & 0.44 & 0.44 \\
\hline 359 & 2XMM J154316.4+540526 & 0.2452 & 2 & -12.85 & 43.38 & 0.49 & 0.49 \\
\hline 360 & 2XMM J154359.4+535902 & 2.371 & 2 & -13.05 & 45.43 & 0.06 & 0.06 \\
\hline 361 & 2XMM J154530.3+484608 & 0.3993 & 4 & -12.16 & 44.55 & 0.15 & 3.06 \\
\hline 362 & 2XMM J154535.8+484713 & 1.404 & 3 & -13.34 & 44.63 & 0.40 & 2.82 \\
\hline 363 & 2XMM J160318.0+430116 & 1.156 & 2 & -13.42 & 44.36 & -0.04 & -0.04 \\
\hline 364 & 2XMM J160419.0+325631 & 2.281 & 2 & -13.46 & 44.98 & 2.06 & 2.06 \\
\hline 365 & 2XMM J160501.3+174515 & 2.996 & 2 & -13.75 & 44.95 & -0.36 & -0.36 \\
\hline 366 & 2XMM J160513.1+325829 & 2.256 & 5 & -13.57 & 44.87 & 0.09 & 2.10 \\
\hline 367 & 2XMM J160603.6+174307 & 1.105 & 2 & -13.58 & 44.15 & -0.08 & -0.08 \\
\hline 368 & 2XMM J160613.5+325554 & 1.874 & 6 & -13.57 & 44.68 & -0.17 & 2.72 \\
\hline 369 & 2XMM J161706.8+122606 & 1.637 & 2 & -13.36 & 44.76 & -1.12 & -1.12 \\
\hline 370 & 2XMMi J162710.3+350118 & 2.288 & 2 & -13.39 & 45.05 & -0.21 & -0.21 \\
\hline 371 & 2XMMi J162722.4+351039 & 1.677 & 2 & -13.31 & 44.83 & -0.12 & -0.12 \\
\hline 372 & 2XMM J162855.6+394034 & 1.521 & 3 & -13.30 & 44.75 & -0.11 & 1.22 \\
\hline 373 & 2XMM J162937.1+394059 & 0.7241 & 3 & -13.08 & 44.23 & 0.05 & 1.38 \\
\hline 374 & 2XMM J162940.4+393124 & 2.146 & 3 & -13.50 & 44.89 & -0.21 & 1.12 \\
\hline 375 & 2XMMi J163023.5+242546 & 2.312 & 3 & -13.85 & 44.61 & -0.22 & 0.38 \\
\hline 376 & 2XMM J164056.2+363404 & 0.6761 & 2 & -12.42 & 44.81 & 0.08 & 0.08 \\
\hline 377 & 2XMM J165430.7+395418 & 0.3397 & 2 & -12.39 & 44.16 & 0.17 & 0.17 \\
\hline 378 & 2XMM J165713.2+352441 & 2.331 & 6 & -13.86 & 44.61 & -0.22 & 2.52 \\
\hline 379 & 2XMM J170554.0+240638 & 0.9021 & 2 & -13.27 & 44.25 & 2.29 & 2.29 \\
\hline 380 & 2XMM J170606.2+240305 & 0.7912 & 3 & -13.40 & 43.99 & 1.94 & 2.31 \\
\hline 381 & 2XMM J170639.3+240606 & 0.8358 & 2 & -13.44 & 44.01 & 1.93 & 1.93 \\
\hline 382 & 2XMMi J171029.2+590833 & 0.8637 & 2 & -12.87 & 44.61 & 0.87 & 0.87 \\
\hline 383 & 2XMMi J171126.8+585543 & 0.5373 & 2 & -12.77 & 44.23 & 0.96 & 0.96 \\
\hline 384 & 2XMMi J171144.9+584917 & 1.533 & 2 & -13.16 & 44.89 & 0.74 & 0.74 \\
\hline 385 & 2XMM J171359.4+640939 & 1.36 & 2 & -13.52 & 44.42 & 1.29 & 1.29 \\
\hline 386 & 2XMMi J171815.9+584613 & 1.413 & 6 & -13.38 & 44.59 & -0.08 & 1.92 \\
\hline 387 & 2XMMi J171818.1+584904 & 0.6344 & 7 & -13.10 & 44.07 & 0.09 & 2.09 \\
\hline 388 & 2XMMi J171930.2+584804 & 2.081 & 8 & -13.05 & 45.30 & -0.19 & 1.82 \\
\hline 389 & 2XMMi J172026.4+263816 & 1.145 & 3 & -13.56 & 44.20 & -0.04 & 0.27 \\
\hline 390 & 2XMMi J172052.1+590154 & 0.3512 & 8 & -12.77 & 43.81 & 0.17 & 2.17 \\
\hline 391 & 2XMMi J172130.9+584405 & 0.9997 & 7 & -12.72 & 44.91 & 0.00 & 2.00 \\
\hline 392 & 2XMM J172255.3+320307 & 0.2752 & 3 & -12.90 & 43.44 & 0.79 & 1.24 \\
\hline 393 & 2XMM J172256.7+321427 & 1.173 & 3 & -12.83 & 44.95 & 0.56 & 1.00 \\
\hline 394 & 2XMMi J172310.4+595105 & 0.9899 & 2 & -13.33 & 44.29 & 0.47 & 0.47 \\
\hline 395 & 2XMMi J172353.2+600002 & 1.453 & 2 & -13.09 & 44.91 & 0.38 & 0.38 \\
\hline
\end{tabular}


A\&A 536, A84 (2011)

Table 1. continued.

\begin{tabular}{lccccccc}
\hline \hline $\begin{array}{l}N_{\text {sou }} \\
(1)\end{array}$ & $\begin{array}{c}\text { Name } \\
(2)\end{array}$ & $\begin{array}{c}z \\
(3)\end{array}$ & $\begin{array}{c}N_{\text {epo }} \\
(4)\end{array}$ & $\begin{array}{c}\log f_{\mathrm{X}} \\
(5)\end{array}$ & $\begin{array}{c}\log L_{\mathrm{X}} \\
(6)\end{array}$ & $\begin{array}{c}\log \tau_{\min } \\
(7)\end{array}$ & $\begin{array}{c}\log \tau_{\max } \\
(8)\end{array}$ \\
\hline 396 & 2XMM J212912.1+120750 & 1.149 & 4 & -13.50 & 44.27 & 1.90 & 2.64 \\
397 & 2XMM J215703.7-073829 & 1.899 & 2 & -13.39 & 44.87 & 2.28 & 2.28 \\
398 & 2XMM J221640.1+001619 & 1.019 & 2 & -13.47 & 44.18 & 1.14 & 1.14 \\
399 & 2XMM J221708.9+002718 & 1.112 & 2 & -13.20 & 44.53 & 1.13 & 1.13 \\
400 & 2XMM J221715.1+002615 & 0.7532 & 2 & -13.10 & 44.24 & 1.21 & 1.21 \\
401 & 2XMM J221738.4+001207 & 1.121 & 2 & -13.76 & 43.99 & 1.12 & 1.12 \\
402 & 2XMM J221739.2+002903 & 1.646 & 2 & -14.21 & 43.91 & 1.03 & 1.03 \\
403 & 2XMM J221751.3+001146 & 1.491 & 2 & -13.64 & 44.39 & 1.05 & 1.05 \\
404 & 2XMM J221755.2+001513 & 2.092 & 2 & -13.90 & 44.46 & 0.96 & 0.96 \\
405 & 2XMM J221806.6+000534 & 2.276 & 2 & -13.95 & 44.49 & 0.93 & 0.93 \\
406 & 2XMM J231733.6+001129 & 0.8407 & 2 & -13.29 & 44.16 & 1.98 & 1.98 \\
407 & 2XMM J231742.5+000535 & 0.3209 & 2 & -12.26 & 44.23 & 2.13 & 2.13 \\
408 & 2XMM J231850.6+002554 & 1.592 & 2 & -13.38 & 44.71 & 2.81 & 2.81 \\
409 & 2XMMi J232810.5+150012 & 1.536 & 2 & -13.64 & 44.42 & 2.33 & 2.33 \\
410 & 2XMMi J235800.6-000107 & 1.454 & 2 & -13.18 & 44.83 & 1.91 & 1.91 \\
411 & 2XMMi J235844.9-000723 & 1.979 & 2 & -13.52 & 44.79 & 1.82 & 1.82 \\
412 & 2XMMi J235845.6-000458 & 1.609 & 2 & -13.24 & 44.86 & 1.88 & 1.88 \\
\hline
\end{tabular}


Table 2. Swift sample.

\begin{tabular}{lcccccc}
\hline \hline $\begin{array}{l}N_{\text {sou }} \\
(1)\end{array}$ & $\begin{array}{c}\text { Name } \\
(2)\end{array}$ & $\begin{array}{c}N_{\text {bin }} \\
(3)\end{array}$ & $\begin{array}{c}\log f_{\mathrm{X}} \\
(5)\end{array}$ & $\begin{array}{c}\log L_{\mathrm{X}} \\
(6)\end{array}$ & $\begin{array}{c}\text { GRB field } \\
(7)\end{array}$ \\
\hline 1 & SWIFTFT J005503+1408.0 & 1.67 & 14 & -13.17 & 45.39 & GRB050904 \\
2 & SWIFTFT J020643+0023.7 & 1.22 & 15 & -13.60 & 44.85 & GRB060908 \\
3 & SWIFTFT J020710+0010.3 & 0.92 & 16 & -13.62 & 44.37 & GRB060908 \\
4 & SWIFTFT J020727+0028.9 & 1.18 & 12 & -13.53 & 44.65 & GRB060908 \\
5 & SWIFTFT J075122+3109.8 & 1.31 & 24 & -13.44 & 44.77 & GRB070125 \\
6 & SWIFTFT J082057+3153.9 & 1.07 & 13 & -12.72 & 45.26 & GRB051227 \\
7 & SWIFTFT J084819+1336.1 & 1.48 & 22 & -13.02 & 45.35 & GRB051016B \\
8 & SWIFTFT J092733+3022.7 & 1.34 & 8 & -13.42 & 45.04 & GRB050505 \\
9 & SWIFTFT J092736+3020.0 & 1.26 & 12 & -12.94 & 45.28 & GRB050505 \\
10 & SWIFTFT J094821+3153.9 & 1.59 & 10 & -13.43 & 45.07 & GRB060108 \\
11 & SWIFTFT J101609+4336.2 & 0.59 & 21 & -12.80 & 44.59 & GRB050319 \\
12 & SWIFTFT J101727+4329.0 & 1.17 & 17 & -13.10 & 45.00 & GRB050319 \\
13 & SWIFTFT J113805+4047.5 & 2.18 & 8 & -13.13 & 45.62 & GRB050215B \\
14 & SWIFTFT J114502+5957.3 & 1.64 & 75 & -13.39 & 45.06 & GRB060319 \\
15 & SWIFTFT J120215+1045.3 & 1.32 & 29 & -13.11 & 45.07 & GRB050408 \\
16 & SWIFTFT J121017+3956.7 & 0.40 & 15 & -12.74 & 44.20 & GRB070419A \\
17 & SWIFTFT J121645+3529.6 & 2.01 & 21 & -13.39 & 45.33 & GRB060712 \\
18 & SWIFTFT J124958+3028.8 & 1.63 & 5 & -13.13 & 45.28 & GRB050520 \\
19 & SWIFTFT J131524+1638.0 & 1.44 & 19 & -13.64 & 44.66 & GRB070406 \\
20 & SWIFTFT J133128+4209.7 & 0.94 & 11 & -13.67 & 44.11 & GRB051008 \\
21 & SWIFTFT J140704+2735.8 & 2.22 & 17 & -13.22 & 45.55 & GRB060204B \\
22 & SWIFTFT J141221+1657.9 & 1.87 & 7 & -13.26 & 45.32 & GRB060801 \\
23 & SWIFTFT J143646+2745.0 & 0.22 & 19 & -13.50 & 42.43 & GRB050802 \\
24 & SWIFTFT J144339+1229.4 & 1.98 & 5 & -13.27 & 45.31 & GRB060805 \\
25 & SWIFTFT J144419+1236.3 & 1.51 & 6 & -13.49 & 44.95 & GRB060805 \\
26 & SWIFTFT J160757+3221.8 & 1.42 & 12 & -13.52 & 44.81 & GRB060219 \\
27 & SWIFTFT J165004+3133.9 & 1.70 & 11 & -13.50 & 44.98 & GRB060807 \\
\hline & & & & & &
\end{tabular}

